Check for updates

Cite this: DOI: 10.1039/c9ta06781g

\title{
Crystal size versus paddle wheel deformability: selective gated adsorption transitions of the switchable metal-organic frameworks DUT-8(Co) and DUT-8(Ni) $\uparrow$
}

\author{
Sebastian Ehrling, (D) ${ }^{a}$ Irena Senkovska, (D) *a Volodymyr Bon, (D) a Jack D. Evans, (DD ${ }^{a}$ \\ Petko Petkov, (D) ${ }^{\mathrm{b}}$ Yulia Krupskaya, ${ }^{c}$ Vladislav Kataev, (D) ${ }^{\mathrm{c}}$ Toshiki Wulf, (D) de \\ Alexander Krylov, (D) ${ }^{f}$ Alexander Vtyurin, (D) fg Svetlana Krylova, (D) f Sergey Adichtchev, ${ }^{\text {h }}$ \\ Evgenia Slyusareva, (D) 9 Manfred S. Weiss, (D) i Bernd Büchner, (D) ${ }^{c}$ Thomas Heine (D) ej \\ and Stefan Kaskel (DD *a
}

\begin{abstract}
Switchable pillared layer metal-organic frameworks $M_{2}(2,6-n d c)$ (dabco) (DUT-8(M), $M=N i, C o, 2,6-n d c=2,6$ naphthalenedicarboxylate, dabco = 1,4-diazabicyclo-[2.2.2] octane, DUT - Dresden University of Technology) were synthesised in two different crystallite size regimes to produce particles up to $300 \mu \mathrm{m}$ and smaller particles around $0.1 \mu \mathrm{m}$, respectively. The textural properties and adsorption-induced switchability of the materials, obtained from both syntheses, were studied by physisorption of $\mathrm{N}_{2}$ at $77 \mathrm{~K}, \mathrm{CO}_{2}$ at $195 \mathrm{~K}$ and $n$ butane at $273 \mathrm{~K}$, revealing pronounced differences in adsorption behavior for $\mathrm{Ni}$ and $\mathrm{Co}$ analogues. While the smaller nano-sized particles (50-200 nm) are rigid and show no gating transitions confirming the importance of crystallite size, the large particles show pronounced switchability with characteristic differences for the two metals resulting in distinct recognition effects for various gases and vapours. Adsorption of various vapours demonstrates consistently a higher energetic barrier for the "gate opening" of DUT-8(Co) in contrast to DUT$8(\mathrm{Ni})$, as the "gate opening" pressure for Co based material is shifted to a higher value for adsorption of dichloromethane at $298 \mathrm{~K}$. Evaluation of crystallographic data, obtained from single crystal and powder X-ray diffraction analysis, showed distinct geometric differences in the paddle wheel units of the respective MOFs. These differences are further disclosed by solid-state UV-vis, FT-IR and Raman spectroscopy. Magnetic properties of DUT-8(Co) and DUT-8(Ni) were investigated, indicating a high-spin state for both materials at room temperature. Density functional theory (DFT) simulations confirmed distinct energetic differences for $\mathrm{Ni}$ and Co analogues with a higher energetic penalty for the structural "gate opening" transformation for DUT8(Co) compared to DUT-8(Ni) explaining the different flexibility behaviour of these isomorphous MOFs.
\end{abstract}

Received 24th June 2019

Accepted 1st September 2019

DOI: $10.1039 /$ c9ta06781g

rsc.li/materials-a

\section{Introduction}

Beyond ultrahigh porosity and modular pore structure, switchable porosity is a unique feature of some metal-organic frameworks (MOFs) attracting extensive research interest ${ }^{1-8}$ especially after the pioneering work by Kitagawa and Férey. ${ }^{9,10}$

We consider switchable porosity as a cooperative, step-wise structural transformation induced by physical or chemical stimuli resulting in dynamic pore expansion or shrinkage. The
${ }^{a}$ Chair of Inorganic Chemistry, Technische Universität Dresden, Bergstrasse 66, 01069 Dresden, Germany.E-mail: irena.senkovska@tu-dresden.de; stefan.kaskel@ tu-dresden.de

${ }^{b}$ University of Sofia, Faculty of Chemistry and Pharmacy, 1126, Sofia, Bulgaria ${ }^{c}$ Leibniz Institute for Solid State and Materials Research, IFW Dresden, Helmholtzstrasse 20, 01069 Dresden, Germany

${ }^{d}$ Wilhelm-Ostwald-Institute of Physical and Theoretical Chemistry, Faculty for Chemistry and Mineralogy, Leipzig University, 04103 Leipzig, Germany

${ }^{e}$ Helmholtz-Zentrum Dresden-Rossendorf, Institute of Resource Ecology, Research Site Leipzig, Permoserstr. 15, 04318 Leipzig, Germany

${ }^{f}$ Kirensky Institute of Physics, Federal Research Center KSC SB RAS, 660036 Krasnoyarsk, Russia

${ }^{g}$ Siberian Federal University, Svobodny Prospect 79, 660041 Krasnoyarsk, Russia
${ }^{h}$ Institute of Automation and Electrometry of the SB RAS, 630090, Novosibirsk, Russia

${ }^{i}$ Research Group Macromolecular Crystallography, Helmholtz-Zentrum Berlin für Materialien und Energie, Albert-Einstein-Straße 15, 12489 Berlin, Germany

${ }^{j}$ Chair of Theoretical Chemistry, Technische Universität Dresden, Berg-strasse 66, 01069 Dresden, Germany

$\dagger$ Electronic supplementary information (ESI) available: Rietveld plots, SEM images, thermogravimetric analysis, gas adsorption data, PXRD patterns, vapour adsorption data, heat of adsorption determination, crystallographic figures, IR spectra, Raman spectra, spin related cluster and framework optimization, simulated UV-vis spectra. CCDC 1912199, 1912198 and 1912233. For ESI and crystallographic data in CIF or other electronic format see DOI: 10.1039/c9ta06781g 
underlying structural phase transitions are triggered by adsorption or desorption of guest molecules and generally characterised by an activation energy barrier, which causes hysteresis in physisorption experiments. Due to their switchable nature, soft porous crystals are often discussed as materials with huge application potential in gas storage, ${ }^{\mathbf{1 1}}$ separation processes, ${ }^{\mathbf{1 2 , 1 3}}$ sensor technology ${ }^{\mathbf{1 4 , 1 5}}$ and catalysis. ${ }^{\mathbf{1 6}}$

Despite rapidly growing research in the field of flexible MOFs, ${ }^{\mathbf{1 1 7 - 2 1}}$ the role of critical factors influencing and controlling framework switchability are barely understood. ${ }^{22}$ As MOFs are modular networks, the flexibility of the linker but also the hinges of the metal node are key features that affect switchability. The importance of metal-node hinges and their energetics for framework switchability has been widely investigated for compounds such as $\mathrm{M}(\mathrm{bdp})(\mathrm{M}=\mathrm{Co}, \mathrm{Fe}$, bdp $=1,4$-benzenedipyrazolate), ${ }^{11,23,24} \mathrm{M}(\mu-\mathrm{OH})(\mathrm{bdc})(\mathrm{MIL}-53, \mathrm{M}=\mathrm{Cr}$, Al, Fe, bdc $=1,4$-benzenedicarboxylate $)^{25-27}$ and pillared layer MOFs with the general formula $\mathrm{M}_{2} \mathrm{~L}_{2} \mathrm{P},(\mathrm{L}=$ ditopic carboxylate, $\mathrm{P}=$ neutral pillar). ${ }^{1728,29}$ In pillared layer MOFs, binuclear paddle wheel nodes $\mathrm{M}_{2}(\mathrm{COO})_{4}$ are connected by ditopic carboxylates to form 2D grids, which are pillared by neutral diamine donor ligands to form a 3D framework with pcu topology. Several pillared layer MOFs show some degree of framework flexibility and they can be synthesised using a variety of transition metals, such as Co, $\mathrm{Ni}, \mathrm{Cu}$, and $\mathrm{Zn}$, rendering them an ideal model system to achieve a deeper understanding of framework switchability. ${ }^{30-32}$

One of the first representatives of this family, $\mathrm{Cu}_{2}(\mathrm{bdc})_{2}$ dabco (dabco = 1,4-diazabicyclo-[2.2.2]-octane), was synthesised by Seki et al. in 2002 (ref. 33), followed by report on $\mathrm{Zn}_{2}$ (bdc) ${ }_{2}$ dabco, in 2004 by Dybtsev et al., ${ }^{34} \mathrm{Co}_{2}$ (bdc) ${ }_{2}$ dabco by Kaskel and co-workers in 2008 (ref. 35) and $\mathrm{Ni}_{2}$ (bdc) ${ }_{2}$ dabco by Stock and co-workers in 2011. ${ }^{36}$ These compounds show contractive/expansive behaviour during desolvation/resolvation of the framework but do not show pronounced switchability upon gas adsorption. Connecting $\mathrm{Ni}, \mathrm{Cu}$, $\mathrm{Co}$ or $\mathrm{Zn}$ paddle wheels by 2,6-naphtalenedicarboxylates and dabco molecules, resulted in isoreticular frameworks, further denoted as DUT$8(\mathrm{M})$ (DUT $=$ Dresden University of Technology, $\mathrm{M}=\mathrm{Ni}$, Co, $\mathrm{Cu}$ and $\mathrm{Zn}) .{ }^{37}$ The nickel-based compound shows pronounced stimuli-induced "gating"-type flexibility upon adsorption of gases and liquids. ${ }^{38}$ In situ ${ }^{129} \mathrm{Xe}$ NMR and powder X-ray diffraction (PXRD) were used to elucidate the guest-framework interactions, framework dynamics and crystal structure of the closed pore phase, indicating an unprecedented unit cell volume change of $250 \%$ upon "gate opening". 39

Recent studies revealed that not only the chemical composition and average crystal structure, but also the real particle structure (size, morphology, defects) considerably affects the switchability, increasing the complexity of investigations. ${ }^{40,41}$ In fact, for smaller crystals $(d<500 \mathrm{~nm})$ switchability was entirely suppressed $^{\mathbf{4 0}}$ and these crystals also contain a higher concentration of defects. ${ }^{42}$ This complexity has motivated us to reinvestigate the importance of various metal nodes, while controlling the crystallite size, for switchable MOFs. This allows us to independently analyse cooperative coupling versus metal node flexibility.
In the following, we analyse the cobalt based DUT-8 compound (DUT-8(Co)), isomorphous to DUT-8(Ni), which shows pronounced differences in the adsorption behaviour compared to the nickel-based counterpart. We show activation barriers of the opening transition of DUT-8(Co) to be higher than for DUT-8(Ni) while the energetic differences of $o p$ and $c p$ forms are less distinct. Moreover, the switching behaviour is controlled by crystal (size) engineering, an effect superimposed on the effect of the metal node.

\section{Experimental section}

\section{General}

$\mathrm{Co}\left(\mathrm{NO}_{3}\right)_{2} \cdot 6 \mathrm{H}_{2} \mathrm{O} \quad(\geq 98 \%), \quad \mathrm{Ni}\left(\mathrm{NO}_{3}\right)_{2} \cdot 6 \mathrm{H}_{2} \mathrm{O}$ ( $\left.\geq 98 \%\right), \quad 2,6-\mathrm{H}_{2}$ ndc (99\%), dabco were purchased from Sigma Aldrich. All solvents were at least of analytical grade, purchased from commercial suppliers and used without further purification.

Scanning electron microscopy (SEM) measurements were carried out using $2 \mathrm{kV}$ acceleration voltage and a working distance of $14 \mathrm{~mm}$ on a SU8020 from Hitachi. Powder X-ray diffraction (PXRD) experiments at room temperature were carried out using STOE STADI P diffractometer with $\mathrm{Cu}-\mathrm{K} \alpha 1$ radiation $(\lambda=1.54059 \AA$ ) and a 2D detector (Mythen, Dectris) in transmission geometry. For gas and vapour adsorption measurements Belsorp Max volumetric adsorption instrument from MicrotracBEL Corp. (Japan) was used. Prior to the measurements, the samples were desolvated in dynamic vacuum at room temperature for at least 16 hours. UV/Vis experiments were carried out at $25^{\circ} \mathrm{C}$ using a Cary 4000 (Varian) equipped with a praying mantis holder in diffuse reflection mode between 400 and $800 \mathrm{~nm}$ with a step of $0.5 \mathrm{~nm}, \mathrm{BaSO}_{4}$ was used as filling material. FT-IR spectra were recorded on a Bruker Vertex 70 in the range from 4000 to $400 \mathrm{~cm}^{-1}$ (resolution $2 \mathrm{~cm}^{-1}$, ATR). Elemental analyses were performed on a Hekatech EA 3000 Euro Vector CHNS. TG-DTA was carried out using a STA 409 (Netzsch) from room temperature to $800{ }^{\circ} \mathrm{C}$ (heating rate of $5 \mathrm{~K} \mathrm{~min}^{-1}$ under synthetic air).

\section{MOF synthesis}

An overview of all samples and their general switching characteristics are summarised in Scheme 1.

DUT-8(Co)_macro-sized, 1a_op. Typically, $\mathrm{Co}\left(\mathrm{NO}_{3}\right)_{2} \cdot 6 \mathrm{H}_{2} \mathrm{O}$

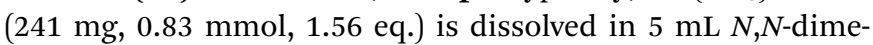
thylformamide (DMF), 2,6- $\mathrm{H}_{2}$ ndc $(178.5 \mathrm{mg}, 0.83 \mathrm{mmol}, 1.56$ eq.) is dissolved $15 \mathrm{~mL} \mathrm{DMF}$ and dabco (60 $\mathrm{mg}, 0.53 \mathrm{mmol}$ ) is dissolved in $5 \mathrm{~mL}$ DMF.

The three resulting solutions are combined in a Teflon vessel $(50 \mathrm{~mL})$ and heated in an autoclave at $120^{\circ} \mathrm{C}$ for 48 hours. The resulting dark blue crystals were washed with DMF several times.

DUT-8(Co)_macro-sized, 1a_cp. Samples of 1a_op were soaked in dichloromethane (DCM) and the supernatant solution was replaced several times in the first two hours. After two days, the crystals were filtered under argon flow and the solvent was removed under vacuum overnight at room temperature (RT). During solvent removal, the colour of the sample changed 


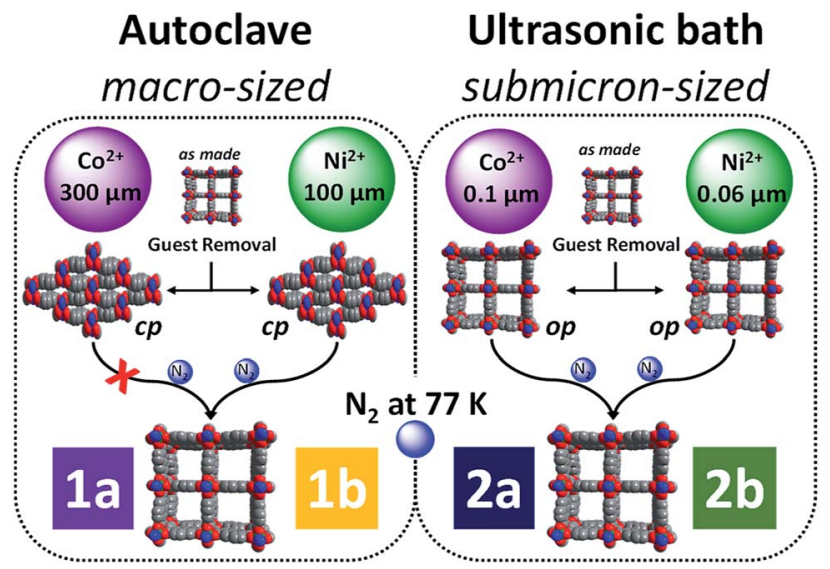

Scheme 1 Schematic overview of the samples investigated: DUT-8(M) ( $\mathrm{M}: \mathrm{Ni}, \mathrm{Co})$ were synthesised, using different precursor concentrations yielding products of varying crystal sizes. Marco-sized crystals of DUT$8(M)$ undergo structural transformation during solvent removal, whereas the submicron-sized particles remain in an open pore state. Furthermore, a reopening of the structure of DUT-8(Co)_macro-sized $\left(1 \mathrm{a} \_c p\right)$ with $\mathrm{N}_{2}$ at $77 \mathrm{~K}$ is not possible.

from dark blue to dark purple. Yield: $263 \mathrm{mg}, 96 \%$ (based on $\left.\mathrm{Co}^{2+}\right)$. The phase purity of the product $1 \mathrm{a}$ was determined by CHN analysis: $\mathrm{C}_{30} \mathrm{H}_{26} \mathrm{O}_{9} \mathrm{~N}_{2} \mathrm{Co}_{2}$ : calc.: $\mathrm{C}, 53.478 ; \mathrm{H}, 3.874 ; \mathrm{N}$, 4.142; found: C, $53.96 \pm 0.07 ; \mathrm{H}, 3.585 \pm 0.151 ; \mathrm{N}, 4.95 \pm 0.02$.

DUT-8(Ni)_macro-sized, 1b_op. The samples were prepared according to the synthetic procedure reported in ref. 41.

DUT-8(Ni)_macro-sized, 1b_cp. Samples of 1b_op were soaked in DCM and the supernatant solution was replaced several times in the first two hours. After two days, the crystals were filtered under argon flow and the solvent was removed under vacuum overnight at room temperature (RT). During solvent removal, the colour of the sample changed from green to yellow.

DUT-8(Co)_submicron-sized, 2a_as made. Typically, 2,6$\mathrm{H}_{2}$ ndc (178.5 mg, $0.83 \mathrm{mmol}$ ) was dissolved in $25 \mathrm{~mL}$ DMF. Once the solution was clear $\mathrm{Co}\left(\mathrm{NO}_{3}\right)_{2} \cdot 6 \mathrm{H}_{2} \mathrm{O}(241 \mathrm{mg}, 0.83$ $\mathrm{mmol}$ ) was added. Subsequently, dabco (300 mg, $2.67 \mathrm{mmol}, 3.2$ eq.) was added whereby the solution immediately turned cloudy. The resulting mixture was then treated in an ultra-sonic bath for 60 minutes increasing the suspension temperature from $25{ }^{\circ} \mathrm{C}$ to $55{ }^{\circ} \mathrm{C}$. The mother solution was removed by centrifugation and the resulting particles were washed several times with DMF.

DUT-8(Co)_submicron-sized, 2a_op. This work-up is identical to that for the flexible compound 1a. A colour change during solvent removal was not observed, i.e. the particles remain greyish-blue.

Yield: $245 \mathrm{mg}, 90 \%$ (based on $\mathrm{Co}^{2+}$ ). The phase purity of the product $2 \mathrm{a}$ was determined by $\mathrm{CHN}$ analysis: $\mathrm{C}_{30} \mathrm{H}_{26} \mathrm{O}_{9} \mathrm{~N}_{2} \mathrm{Co}_{2}$ : calc.: C, 53.478; H, 3.874; N, 4.142; found: C, $54.45 \pm 0.38 ; \mathrm{H}$, $3.870 \pm 0.074 ; \mathrm{N}, 4.46 \pm 0.02$.

DUT-8(Ni)_submicron-sized, 2b_as made. Typically, 2,6$\mathrm{H}_{2}$ ndc (296 mg, $1.37 \mathrm{mmol}$ ) was dissolved in $30 \mathrm{~mL}$ DMF. Once the solution was clear, $\mathrm{Ni}\left(\mathrm{NO}_{3}\right)_{2} \cdot 6 \mathrm{H}_{2} \mathrm{O}(436.2 \mathrm{mg}, 1.5 \mathrm{mmol}, 1.1$ eq.) was added. Finally, dabco (337 mg, $3 \mathrm{mmol}, 2.2$ eq.) was added, whereby the solution immediately turned cloudy. The resulting mixture was then treated in an ultrasonic bath for 60 minutes increasing the suspension temperature from $25{ }^{\circ} \mathrm{C}$ to $55{ }^{\circ} \mathrm{C}$. The mother solution was removed by centrifugation and the resulting particles were washed several times with DMF.

DUT-8(Ni)_submicron-sized, 2b_op. The work-up and handling were identical to that described above for $\mathbf{2 a} \_$op .

\section{Single-crystal X-ray diffraction of 1a_op and 1b_op}

The mother liquor containing single crystals of 1a_op and 1b_op, obtained in the solvothermal synthesis was exchanged to DCM several times in two days. Subsequently, DCM was exchanged to $n$-heptane for 1 day and the crystal was sealed in the glass capillary with a small amount of the solvent. The dataset for DUT-8(Co) was collected at BESSY MX BL14.3 beamline of Helmholtz-Zentrum Berlin für Materialien und Energie. $^{43}$ The monochromatic X-ray radiation with a wavelength of $\lambda=0.8945 \AA(E=13.86 \mathrm{keV})$ was used in the experiment. The room temperature dataset was collected using $\varphi$ scans technique with oscillation range of $1^{\circ}$. Analysis of the crystal symmetry, based on the short scan suggests a monoclinic $C$-centered unit cell for the compound. In order to collect the dataset with maximal completeness, 150 images were measured with $1.2 \mathrm{~s}$ exposure time. The analysis of the reflection datasets suggests a $C$-centered monoclinic group $(C 2 / m)$, different from that reported earlier $(P 4 / n),{ }^{37}$ obtained from Rietveld analysis of PXRD patterns for as made compound in DMF. The datasets were further processed automatically using XDSAPP 2.0 software. ${ }^{44}$ For the DUT-8(Co) structure, the inversion centre, found between two symmetrically dependent nitrogen atoms is not compatible with molecular symmetry of the dabco. Therefore, eight positions of three carbon atoms were refined with occupancies, suggested by the symmetry of the position and involving the factor of disorder. To treat the disorder, the $\mathrm{C}-\mathrm{N}$ and $\mathrm{C}-\mathrm{C}$ distances and thermal parameters within the dabco molecule were treated using DFIX, SIMU and DELU instructions. A low $\left|E^{2}-1\right|$ value, similar $a$ and $b$ parameters and high $R$-values, obtained in the refinement prompt us to check the dataset for pseudo-merohedral twinning using the TwinRotMat program in PLATON. ${ }^{45}$ Indeed, the program suggests a rotation around [110]. As a solution, a new HKLF5 dataset with four domains was generated and used for refinement. This resulted in the improvement of the $R_{1}(\sigma>2)$ value from 0.1989 to 0.0956 for the final refinement cycle. Solvent molecules within the pores of DUT-8(Co) could not be modelled from the difference Fourier map and the SQUEEZE routine could also not be applied because of incompatibility with HKLF5 file format.

The dataset on the DUT-8(Ni) crystal was collected on a Bruker Kappa APEXII diffractometer, equipped with 4-circle goniometer, Mo-K $\alpha$ radiation $(\lambda=0.71073 \AA$, graphite monochromator) and APEX2 CCD-camera. The unit cell parameters were determined based on three short scans, performed on different crystal orientations. The images were integrated using SAINT routine. ${ }^{46}$ Further correction for Lorentz, polarization and absorption effects were performed with the help of 
SADABS software. ${ }^{47}$ Crystal structures were solved by direct methods and refined by full matrix least-squares on $F^{2}$ using SHELX-2016/4 program package ${ }^{48}$ in $C 2 / m$ space group. During the refinement a procedure similar to that applied for DUT$8(\mathrm{Co})$ was used to treat the disorder and twinning in the crystal structure. CCDC-1912199 and -1912198 contain the supplementary crystallographic data for DUT-8(Co) and DUT$8(\mathrm{Ni})$, respectively. $\dagger$

Crystal data for 1a_op ( $\mathrm{Co}_{2}(2,6-\mathrm{ndc})_{2}($ dabco $\left.)(\boldsymbol{n} \text {-heptane })_{\boldsymbol{x}}\right)$. $\mathrm{C}_{30} \mathrm{H}_{24} \mathrm{O}_{8} \mathrm{~N}_{2} \mathrm{Co}_{2}$ (framework only, solvent molecules omitted), $M$ $=658.37 \mathrm{~g} \mathrm{~mol}^{-1}$, monoclinic, $C 2 / m$ (no. 12), $a=18.720(4) \AA$, $b=18.340(4) \AA, c=9.4900(19) \AA, \beta=101.77(3)^{\circ}, V=3189.6(12)$ $\AA^{3}, Z=2, \lambda=0.89495 \AA, T=293 \mathrm{~K}, \theta_{\max }=33.99^{\circ}$, reflections/ parameter $3012 / 118, R_{\text {int }}=0.1308, R_{1}=0.0956, \mathrm{w} R_{2}=0.2862, S$

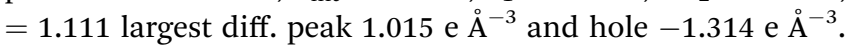

Crystal data for $1 \mathrm{~b} \_o p\left(\mathrm{Ni}_{2}(2,6-n d c)_{2}(\right.$ dabco $\left.)(n \text {-heptane })_{x}\right)$. $\mathrm{C}_{30} \mathrm{H}_{24} \mathrm{O}_{8} \mathrm{~N}_{2} \mathrm{Ni}_{2}$ (framework only, solvent molecules omitted), $M$ $=657.93 \mathrm{~g} \mathrm{~mol}^{-1}$, monoclinic, $C 2 / m$ (no. 12), $a=18.47(2) \AA, b=$ 18.39(2) ̊, $c=9.311(12) \AA, \beta=98.720(15)^{\circ}, V=3125(7) \AA^{3}, Z=$ $2, \lambda=0.71073 \AA, T=293 \mathrm{~K}, \theta_{\max }=26.47^{\circ}$, reflections/parameter $3222 / 109, R_{\text {int }}=0.1644, R_{1}=0.1299, \mathrm{w} R_{2}=0.3910, S=1.239$ largest diff. peak 1.850 e $\AA^{-3}$ and hole -2.844 e $\AA^{-3}$.

\section{Rietveld refinement of $1 \mathrm{a} \_c p$}

It was not possible to preserve the single crystals suitable for SCD, after the solvent removal and "closing", because of the accompanied large volume change. Thus, the crystal structure of 1a_cp was obtained from Rietveld refinement. Highresolution PXRD patterns of DUT-8(Co) were recorded at KMC-2 Beamline of the BESSY II synchrotron operated by Helmholtz-Zentrum Berlin für Materialien und Energie. ${ }^{49}$ The monochromatic radiation with energy of $7300 \mathrm{eV}(\lambda=1.6984 \AA)$ was used in experiment in order to avoid the absorption and $\mathrm{X}$ ray fluorescence on the Co K-edge. An experimental setup, developed for in situ PXRD measurements was used for data collection in transmission geometry. ${ }^{50}$

Since both closed pore phases, DUT-8(Ni) and DUT-8(Co), show very similar PXRD patterns, a structure of DUT-8(Ni)_cp was used as a starting model in the refinement for the Co-based material. The structural model was built in the visualisation module of Materials Studio 5.0 software, ${ }^{51}$ followed by a Pawley fit of the pattern, using Thompson-Cox-Hastings function. The Rietveld refinement was performed in the $P 1$ space group in order to avoid the disorder of the dabco molecule. In order to improve the data/parameter ratio, cobalt atoms, carboxylate groups, naphthalene rings and dabco molecules were treated as rigid bodies. Rietveld refinement with integrated energy contribution of $1 \%$ (based on integrated UFF module) was performed using the Reflex tool of Materials Studio software (Fig. S1, ESI $\dagger$ ). Nine motion groups with 45 degrees of freedom were refined.

Structural data for 1a_cp. $\mathrm{C}_{30} \mathrm{H}_{24} \mathrm{O}_{8} \mathrm{~N}_{2} \mathrm{Co}_{2}, M=658.37 \mathrm{~g}$ mol $^{-1}$, triclinic, $P 1$ (no. 1), $a=7.0454(3) \AA, b=8.2976(4) \AA, c=$ 12.1395(8) A , $\alpha=81.239(7)^{\circ}, \beta=102.217(5)^{\circ}, \gamma=105.558(6)^{\circ}, V$ $=664.75(5) \AA^{3}, Z=1$; zero point line shift $-0.001^{\circ}$; profile function: Thompson-Cox-Hastings, $U=4.71324, V=0.365, W$
$=0.016, X=0.345, Y=-0.109$; asymmetry correction function: Berar-Baldinozzi, $P_{1}=-0.0722, P_{2}=0.002, P_{3}=0.0197, P_{4}=$ 0.0038; global isotropic temperature factor $U=0.01$; refined motion groups 9 , refined degree of freedom $45 ; R_{\mathrm{w}}=12.89 \%, R_{\mathrm{p}}$ $=9.59 \%$.

CCDC-1912233 contains the detailed structural data for 1b_cp. $\dagger$

\section{Raman spectroscopy}

Raman spectra in the $180^{\circ}$ geometry were recorded on a Horiba Jobin Yvon T64000 spectrometer equipped with a liquid nitrogen cooled charge coupled device detection system in subtractive dispersion mode at room temperature. $\mathrm{Ar}^{+}$ion laser Spectra Physics Stabilite 2017 with $\lambda=514.5 \mathrm{~nm}$ and power on the sample of $0.7 \mathrm{~mW}$ was used as an excitation light source. The angular experiments were carried out using the incident laser beam focused on the sample by a $50 \times$ Olympus objective lens with a numerical aperture of 0.75 . The scattered light was collected by the same objective lens and analysed through a polarizer and $\lambda$-plate. Spectroscopic measurements were performed in the subtractive dispersion mode, which attained a low-frequency limit of $10 \mathrm{~cm}^{-1}$ in the present setup, to investigate the low-frequency spectra. The deformation of the low-frequency spectral edge by an optical slit, which sometimes smears the true features of low-frequency spectra, was carefully eliminated by rigorous optical alignment.

\section{Brillouin scattering}

Brillouin scattering experiments were carried out on a six-pass interferometer tandem (JRS Scientific Instruments) at room temperature $(295 \mathrm{~K})$. The solid-state laser Excelsior (Spectra Physics) with $532 \mathrm{~nm}$ wavelength was used to excite Brillouin scattering. The laser power on the sample was about $3 \mathrm{~mW}$. The measurements were carried out in the backscattering geometry without selection by polarisation. The spectra were obtained with several free dispersion regions (FSR) of $30 \mathrm{GHz}$. The spectra of the solvated samples were measured after 15 minutes after the sample was taken from the sealed container in order to evaporate the adhered solvent. The maxima were approximated by means of a fourth degree polynomial interpolation.

\section{Magnetic measurements}

Static magnetization measurements were performed on polycrystalline powder samples using a commercial SQUID (superconducting quantum interference device) VSM (vibrating sample magnetometer) from Quantum Design in a static magnetic field of $1 \mathrm{~T}$ at temperatures between $1.8 \mathrm{~K}$ and $300 \mathrm{~K}$. High-field/high frequency ESR measurements on a polycrystalline powder sample in magnetic fields up to $16 \mathrm{~T}$ for the excitation frequencies from $100 \mathrm{GHz}$ to $280 \mathrm{GHz}$ were done by means of a home-made high-frequency/high-field ESR (HF-ESR) spectrometer based on a vector network analyser (PNA-X from Keysight Technologies) and a $16 \mathrm{~T}$ superconducting magnet system from Oxford Instruments. 


\section{Density functional theory calculations}

Cluster optimisation. Density functional theory (DFT) calculations of the cluster models were performed using the NWchem 6.3 software package ${ }^{52}$ with the PBE0 functional ${ }^{53}$ and a polarized triple-zeta basis set (def2-TZVP) ${ }^{54}$ employing tight convergence criteria. The broken symmetry method was used to model antiferromagnetic coupling for the low-spin (singlet) states. ${ }^{55}$ Input files can be found in the data repository of J. D. Evans [https://github.com/jackevansadl/supp-data].

Periodic model optimization. The calculations of periodic models of DUT-8(Co) MOF were carried out using the QUICKSTEP $^{56,57}$ module of CP2K. ${ }^{58}$ A mixed Gaussian and plane waves basis set with Goedecker-Teter-Hutter (GTH) pseudopotentials ${ }^{59}$ incorporating scalar-relativistic core corrections, named DZVP-MOLOPT-SR-GTH, was used. ${ }^{60-62}$ Periodic boundary conditions were applied in all three dimensions. In our calculations, the REL_CUTOFF parameter was set to $60 \mathrm{Ry}$. The grid cutoff (CUTOFF) of the finest grid level of 360 Ry was used. In all calculations Grimme's DFT-D3 dispersion correction and $\mathrm{DFT}+\mathrm{U}$ approach with $U-J=2.2 \mathrm{eV}$ was applied (for more details see ESI Section $11 \dagger){ }^{63}$

Simulation of UV/vis spectra. UV/vis spectra were calculated with ORCA $^{64}$ using a cluster model derived from the abovementioned periodic structures relaxed at the $\mathrm{PBE}+\mathrm{U}$ level. Both the preparatory constrained geometry optimizations and the TD-DFT parts of these calculations, employed the def2-TZVP basis set and the RIJCOSX ${ }^{65}$ approximation with the $\operatorname{def} 2 / \mathrm{J}$ auxiliary basis set. The broken symmetry approach was used to model antiferromagnetic coupling. To prepare the cluster structures for TD-DFT, the periodic structures were truncated to include one paddle wheel unit with two metal centers, $\mathrm{M}^{2+}$, four 2,6-Hndc ${ }^{-}$linkers and two dabco pillars, saturating dangling bonds with hydrogen atoms. For each metal and spin state, the periodic structure previously obtained for the respective spin state was taken, heavy atoms were frozen in space and the positions of terminal hydrogen atoms were optimized using the PBE0 functional with Grimme's dispersion correction D3(BJ).

TD-DFT on these structures was performed using the CAMB3LYP functional ${ }^{66}$ and making use of the Tamm-Dancoff approximation (TDA). The resulting vertical absorption spectra were broadened using Gaussian functions with a FWHM of $1500 \mathrm{~cm}^{-1}$ and transformed into absorbance spectra by logarithmising the oscillator strength.

\section{Results}

\section{Synthesis and solvent removal}

Considering the crystallite size dependent adsorption-induced switchability, DUT-8(Co) samples were synthesised under controlled conditions (solvothermal vs. high supersaturation). The solvothermal approach results in macro-sized DUT-8(Co) with two distinct crystal size regimes: one around $10 \mu \mathrm{m}$ and the other around $300 \mu \mathrm{m}$. The supersaturation route, however, enables the synthesis of submicron-sized particles (average size $100 \mathrm{~nm}$ ) with a narrow size distribution, according to SEM images (Fig. S2, ESI $\dagger$ ). According to the powder X-ray diffraction
(PXRD) analysis, both synthetic protocols lead to products, isomorphous to the solvated DUT-8(Ni) compound (Fig. 1a and b). To remove the solvent from the pores, $N, N$-dimethylformamide was first exchanged with a low-boiling solvent dichloromethane (DCM), ${ }^{\mathbf{4 1 , 6 7 , 6 8}}$ followed by evacuation at $25{ }^{\circ} \mathrm{C}$. The PXRD patterns of desolvated submicron-sized products, obtained from the supersaturation synthesis, are similar to that of as made materials, indicating the rigidity of submicron-sized products under the applied activation conditions. The PXRD patterns of DUT-8(Ni) and DUT-8(Co) macrocrystals change in the similar manner upon the removal of solvent molecules from the pores, suggesting a similar closing transition pathway for DUT-8(Co) structure, as known for DUT-8(Ni).

Using a controlled solvent exchange protocol, it was possible to obtain phase-pure closed pore form of DUT-8(Co), PXRD patterns of which was used for Rietveld refinement. However, if the macro-sized crystals of DUT-8(Co) were activated directly from DMF, only a phase mixture containing the op and $c p$ phases could be obtained, as reported in 2012 by Klein et al. ${ }^{37}$ Thermogravimetric analysis revealed different thermal stability for macro-sized and submicron-sized particles. 1a is stable up to $360{ }^{\circ} \mathrm{C}$ whilst $\mathbf{1 b}$ shows a reduced stability indicated by a first mass loss at $275^{\circ} \mathrm{C}$ (Fig. S3 and S4, ESI $\dagger$ ).

\section{Adsorption behaviour}

Previous studies have shown that the crystallite size crucially affects the "gate opening" transition of DUT-8(Ni) upon nitrogen adsorption at $77 \mathrm{~K}^{\mathbf{4 0 , 4 1}}$ Three different adsorption characteristics related to the different particle size regimes could be elucidated for DUT-8(Ni): (i) crystallites smaller than $500 \mathrm{~nm}$ remain in the open pore form after removal of the solvent, resulting in a type Ia nitrogen physisorption isotherm; (ii) particles in the range between $500 \mathrm{~nm}$ and $1 \mu \mathrm{m}$ close during solvent removal, but opening with $\mathrm{N}_{2}$ at $77 \mathrm{~K}$ is not possible (type II isotherm); (iii) particles larger than $1 \mu \mathrm{m}$ show flexibility upon adsorption, resulting in typical "gate opening" behavior. ${ }^{\mathbf{4 0}}$

Therefore, the nitrogen adsorption behaviour of submicronand macro-sized crystals of DUT-8(Co) at $77 \mathrm{~K}$ was studied first. 2a show complete accessibility of the pore system, a fully reversible type Ia physisorption isotherm, and $\mathbf{2 b}$ also exhibits similar adsorption behaviour (Fig. 1c).

Surprisingly, crystals of 1a_cp do not undergo any structural change during the adsorption of $\mathrm{N}_{2}$ at $77 \mathrm{~K}$, showing nearly no nitrogen uptake, suggesting no pore accessibility. Obviously, the energy barrier of opening cannot be overcome by adsorption of nitrogen as external stimulus $\left(\Delta H\left(\mathrm{~N}_{2}\right)=-4.1 \mathrm{~kJ} \mathrm{~mol}^{-1}\right.$, infinite dilution from TAP-2 (temporal analysis of products) measurements) ${ }^{69}$ suggesting differences in the energy of "gate opening" for Ni and Co based compounds. The sample 1a even remains in the closed pore form for the whole pressure range during adsorption of $\mathrm{CO}_{2}$ at $195 \mathrm{~K}\left(\Delta H\left(\mathrm{CO}_{2}\right)=-15 \mathrm{~kJ} \mathrm{~mol}^{-1}\right.$, infinite dilution from TAP-2 measurements) and $n$-butane at $273 \mathrm{~K}\left(\Delta H(n\right.$-butane $)=-35 \mathrm{~kJ} \mathrm{~mol}^{-1}$, infinite dilution from TAP-2 measurements) (Fig. S5 and S6, ESI $\dagger$ ). ${ }^{69}$

Higher interaction energies are expected for aprotic polar solvents (such as DMF), producing an opening of the framework 
a)
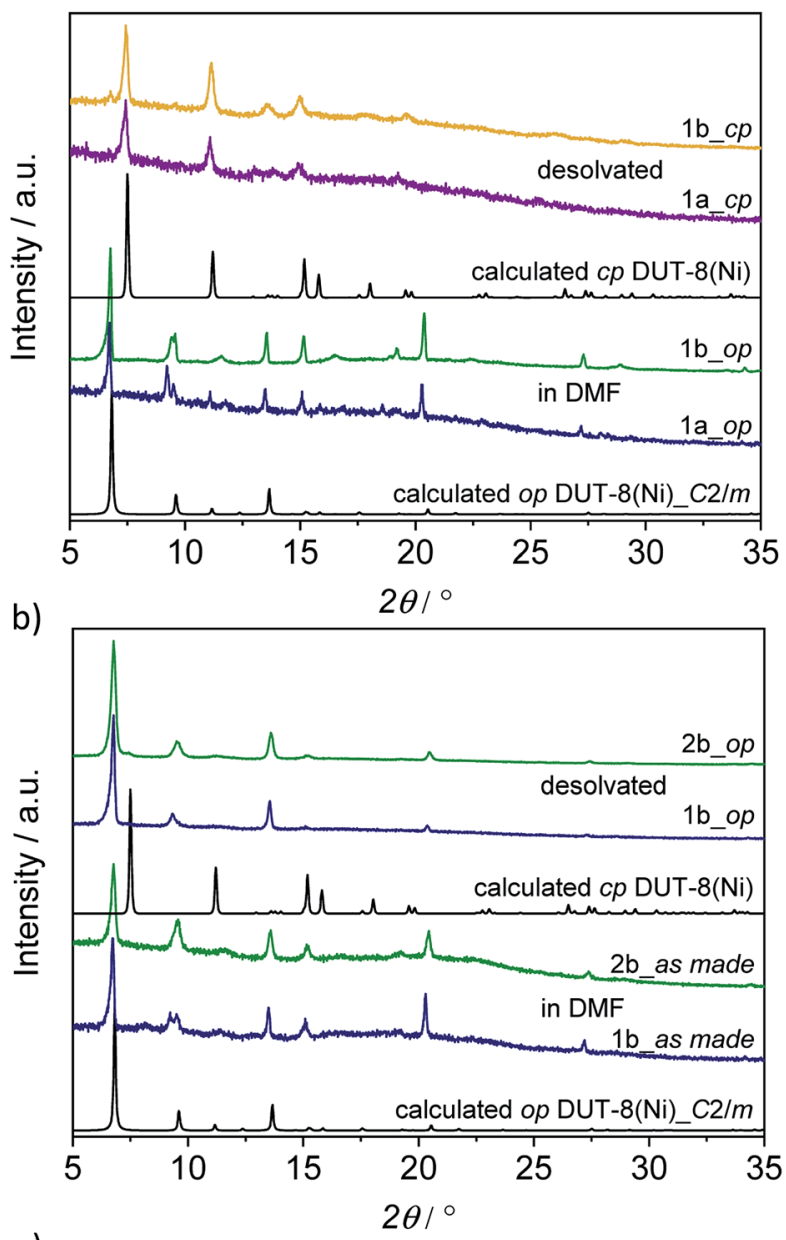

c)

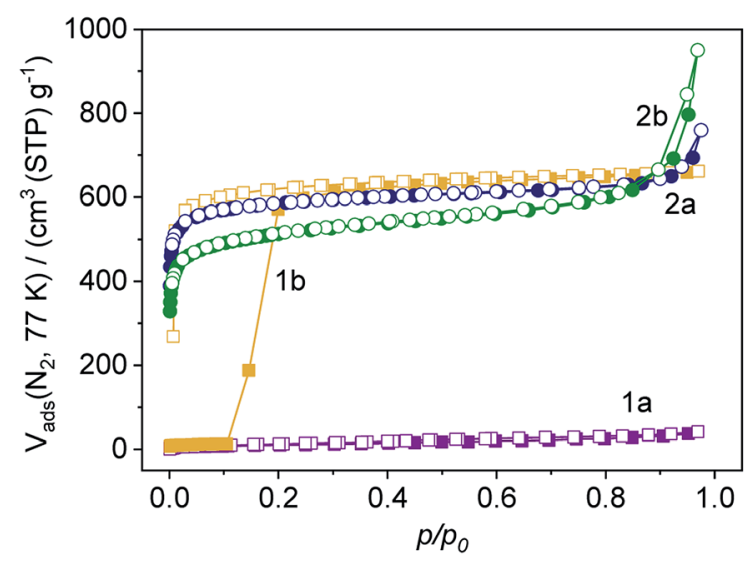

Fig. 1 (a) PXRD patterns of the macro-sized particles after the synthesis (as made) and after solvent removal (desolvated); (b) PXRD patterns of the submicron-sized particles after the synthesis (as made) and after solvent removal (desolvated); (c) respective nitrogen physisorption isotherms of the activated samples at $77 \mathrm{~K}$ (filled symbols adsorption, open symbols - desorption).

independent on particle size for the DUT-8(Ni) system. ${ }^{38}$ Resolvation of 1a_c $c$ in liquid DMF, however, leads only to the opening of part of the sample, according to PXRD analysis
(Fig. S7, ESI $\dagger$ ) suggesting crystal size dependent behaviour. Vapour physisorption experiments using 4 different solvents, including an aprotic non-polar solvent (cyclohexane), an aprotic polar solvent (dichloromethane, DCM) and two protic polar solvents (methanol and ethanol), were performed on 1a_cp. Methanol shows an uptake of nearly $100 \mathrm{mg} \mathrm{g}^{-1}$ at pressures close to saturation, however, this uptake can be attributed to the decomposition of the MOF. These adsorption experiments reveal DCM as the only molecule resulting in a typical "gate opening" isotherm, with "gate opening" at a relative pressure of 0.7 at $298 \mathrm{~K}$ (Fig. 2a). $\mathrm{CHCl}_{3}$ and $\mathrm{CCl}_{4}$ physisorption at $298 \mathrm{~K}$ were also studied, showing a non-flexible response of $\mathbf{1 a} \_p$ for these adsorptives. This is a remarkable finding, as it implies DUT-8(Co) to selectively open its pores only for DCM during the adsorption (Fig. 2b). In contrast, $\mathbf{1 b}$ shows gating behaviour during the adsorption of all chlorinated methanes investigated (Fig. S8, ESI†).

To provide a deeper insight into the interaction energetics, the heats of adsorption of the chlorinated methanes were determined on $\mathbf{2 b} \_o p$. The availability of the "same" MOF structure as rigid and flexible variant provides a unique opportunity for understanding switchability. Adsorption isotherms were recorded at $288 \mathrm{~K}, 298 \mathrm{~K}$ and $308 \mathrm{~K}$ and the Clausius-Clapeyron equation was applied. Since not all isotherms have a classical type Ia shape (see Section 7, ESI †), a fitting with the convention models, such as Langmuir, Freundlich or Dubinin-Radushkevich, produced considerable errors. Thus, linear interpolation was performed to provide equivalent values for the amount adsorbed at various temperatures. The heat of adsorption was calculated as $30-40 \mathrm{~kJ} \mathrm{~mol}^{-1}$ for DCM, 35-50 kJ mol${ }^{-1}$ for chloroform and $25-40 \mathrm{~kJ} \mathrm{~mol}^{-1}$ for tetrachloromethane (Fig. S15, ESI $\dagger$ ). As the dipole moment of $\mathrm{CHCl}_{3}(1.06 \mathrm{D})^{70}$ is significantly smaller than that of DCM $(1.8)^{70}$ a higher interaction is reasonable, as also reported for HKUST$1 .^{71}$ However, the kinetic influence of the respective solvents also needs to be considered. Gate opening from the $c p$ state to the $o p$ state requires overcoming a high energetic barrier. ${ }^{72}$ Hence, due to the larger kinetic diameter of the $\mathrm{CHCl}_{3}(5.47 \AA)^{73}$ molecules compared to DCM $(4.7 \AA),{ }^{73}$ the activation energy for pore entry is expected to be increased. The smaller kinetic diameter of $\mathrm{CH}_{2} \mathrm{Cl}_{2}$ also favours pore opening compared to $\mathrm{CHCl}_{3}$ and therefore the selective opening of 1a_cp for dichloromethane in contrast to other chlorinated methanes is possible.

A direct comparison of the isotherms of $\mathbf{1 a}$ and $\mathbf{1 b}$ (Fig. 2c) shows, that the "gate opening" pressure is shifted to a significantly higher value for DUT-8(Co), implying a higher energetic barrier for "gate opening" for the Co based material, while the closing pressure is almost identical for $\mathrm{Co}$ and $\mathrm{Ni}$ analogues. In addition, the saturation uptake of $1 \mathrm{a}$ is $300 \mathrm{mg} \mathrm{g}^{-1}$ considerably smaller than $\mathbf{1 b}$. This is an indication that at $298 \mathrm{~K}$ the vapour pressure of DCM in the measurement is not sufficient to open all crystals of $\mathbf{1 a}$.

\section{Crystallographic investigations}

Crystal structures of 1a_op. The as-synthesised single crystals of 1a_op containing DMF in the pores show diffuse 
a)

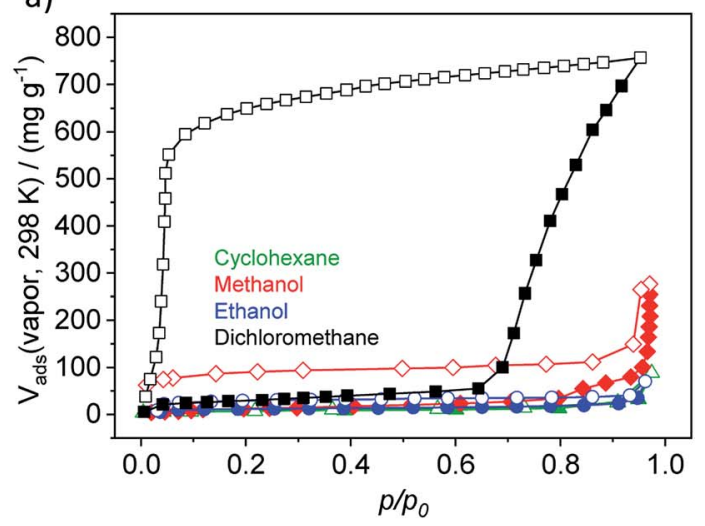

b)

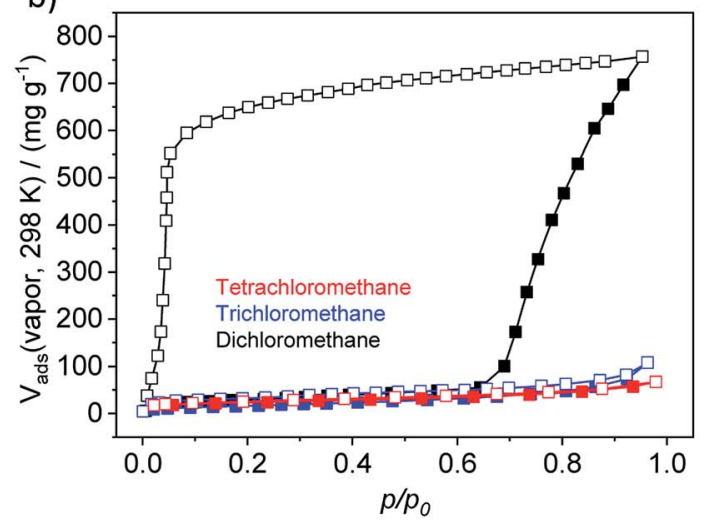

c)

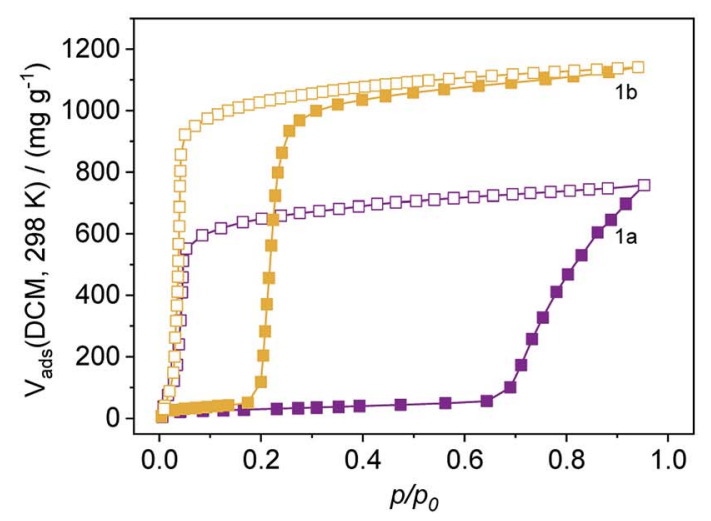

Fig. 2 Vapour physisorption isotherms at $298 \mathrm{~K}$ : (a) for DCM (black), cyclohexane (green), methanol (red) and ethanol (blue) vapours on 1a_cp; (b) chlorinated methanes on 1a_cp, and (c) DCM vapour on $1 a \_c p$ (purple) and 1b_cp (yellow). Filled symbols - adsorption, open symbols - desorption.

scattering effects in the diffraction images, suggesting distinctive deviations from strict crystalline order. ${ }^{74}$ Recent reports ${ }^{75}$ demonstrate that linker disorder in DUT-8(Ni) is solvent dependent. Therefore, to obtain comparable crystallographic data, the solvent in 1a_op and 1b_op crystals was exchanged to $n$-heptane and the data collections were carried out for both compounds under comparable conditions.

Both compounds, containing $n$-heptane in the pores, crystallise in the monoclinic space group $C 2 / m$, different from the
$P 4 / n$ reported earlier for DUT-8(Ni) and DUT-8(Co) originated directly after synthesis in DMF. ${ }^{37,76}$

As expected, the overall crystal structure is based on paddle wheel units, which are interconnected by 2,6-naphthalenedicarboxylates $\left(2,6-\mathrm{ndc}^{2-}\right)$ forming $2 \mathrm{D}$ layers, which are further linked by neutral dabco ligands to form a 3D framework with pcu underlying topology (Fig. 4a).

The main difference between tetragonal and monoclinic polymorphs is the local paddle wheel structure, i.e. the mutual orientation of the quasi-linear 2,6-ndc ${ }^{2-}$ linkers around the Co paddle wheels, as reported in ref. 75 . In the monoclinic structure of DUT-8(M) (M = Ni, Co), the oppositely located 2,6-ndc ${ }^{2-}$ ligands (shown in grey and black in Fig. 4b) point in different directions (up vs. down in respect to the equatorial plane of the paddle wheel).

The nonlinearity of the 2,6-ndc ${ }^{2-}$ molecule is compensated by the tilt of the paddle wheel axes with respect to the $2,6-$ ndc $^{2-}$ $\left(\beta=98.72^{\circ}\right.$ in case of $\mathbf{1 b} \_o p$ and $\beta=101.77^{\circ}$ in case of $\left.\mathbf{1 a} \_o p\right)$. The same arrangement of $2,6-$ ndc $^{2-}$ linkers was found for the majority of the paddle wheel/2,6-ndc ${ }^{2-}$ based crystal structures collected in CCDC data base, $\dagger$ including $\mathrm{Zn},{ }^{77-85} \mathrm{Ni},{ }^{86,87} \mathrm{Co},{ }^{86-88}$ and $\mathrm{Cu}^{89}$ based clusters.

Comparison of monoclinic structures of DUT-8(Ni) and DUT$8(\mathrm{Co})$, filled with $n$-heptane, indicates unit cells with almost equal volumes of 3125(7) and 3190(1) $\AA^{3}$, respectively. The unit cell parameters $a$ and $b$ and ratio between them in DUT-8 structures are responsible for pore accessibility, since the channel like pores are running along $c$ direction (Fig. 4a). In both MOFs there are only minor differences between these parameters and therefore no significant difference in the size of the channels is expected. The more significant difference was found in the $c$ lattice constant, which is 9.311(12) $\AA$ for 1b_op and 9.4900(19) $\AA$ for 1a_op. It correlates with $\mathbf{M} \cdots \mathbf{M}$ spacing
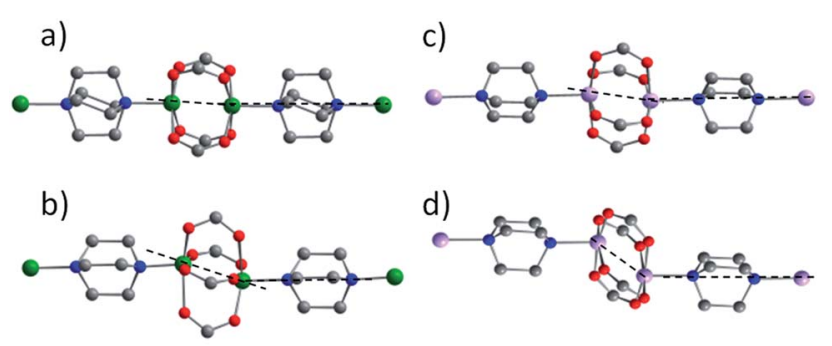

e)

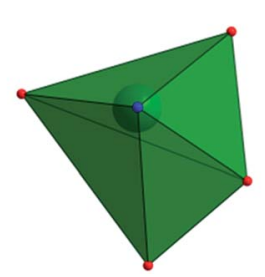

f)

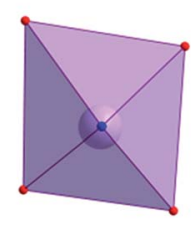

Fig. 3 The alignment of the paddle wheels and pillars in: (a) 1b_op, (b) $1 b \_c p$, (c) 1a_op and (d) 1a_cp. The dashed lines illustrate the angle between the lines running through $\mathrm{N} \cdots \mathrm{N}$ (dabco) and $\mathrm{M} \cdots \mathrm{M}$ (paddle wheel) atoms. (e and f) View on coordination polyhedron of $\mathrm{Ni}$ in 1b_cp (e) and of $\mathrm{Co}$ in 1a_cp (f) along the line connecting $M$ and $N$ atoms. $\mathrm{C}$ - in grey, $\mathrm{O}$ - in red, $\mathrm{N}$ - in blue, $\mathrm{Ni}-$ in green, $\mathrm{Co}-$ in purple. Hydrogen atoms are omitted. 
inside the paddle wheel, $\mathrm{Ni}-\mathrm{N}$ bond lengths, and $\mathrm{N} \cdots \mathrm{N}$ spacing within dabco (Fig. 3a and c).

Thus, the Co $\cdots$ Co distance in 1a_op is about $0.038 \AA$ A longer than $\mathrm{Ni} \cdots \mathrm{Ni}$ distance in $\mathbf{1 b}$ op, which is associated with different ligand-metal bond strength for the two metals. In addition, the $\mathrm{M}-\mathrm{N}$ bond lengths in these two structures differ by $0.068 \AA$ (Table 1). It should also be mentioned that comparison of the coordination geometry of $\mathrm{Ni}$ indicates slightly shorter $\mathrm{M}-\mathrm{O}$ bond lengths, than that in 1a_op. A similar trend is also observed in isoreticular compounds $\mathrm{Ni}_{2}(\mathrm{bdc})_{2} \mathrm{dabco}^{36}$ and $\mathrm{Co}_{2}(\text { bdc })_{2}$ dabco. ${ }^{90}$

The slight difference between the crystal structures of 1b_op and 1a_op is the angle between the $\mathrm{N} \cdots \mathrm{N}$ (dabco) and $\mathrm{M} \cdots \mathrm{M}$ (paddle wheel) axes (Fig. $3 \mathrm{a}$ and c).

In the 1b_op structure, both axes are almost aligned (Ni-Ni$\mathrm{N}$ angle $177.6^{\circ}$ ) while in 1a_op they are tilted with $\mathrm{Co}-\mathrm{Co}-\mathrm{N}$ angle of $171.8^{\circ}$ (Fig. 3a and c). In spite of minor differences in the crystal structures, both op structures show comparable geometrically calculated porosity. Analysis of the pore size distribution in the DUT-8(Ni) and DUT-8(Co) by Zeo++ software $^{91}$ indicates the presence of the channel-like pores of $10.2 \AA$ and $10.0 \AA$ in diameter, respectively.

Crystal structures of 1a_cp. Unfortunately, the single crystals of 1a_cp were not suitable for SCD experiments. Therefore, the crystal structure of 1a_cp phase was refined from PXRD data using Rietveld method. It should be mentioned that PXRD patterns show a broadening of reflections, indicating the

a)

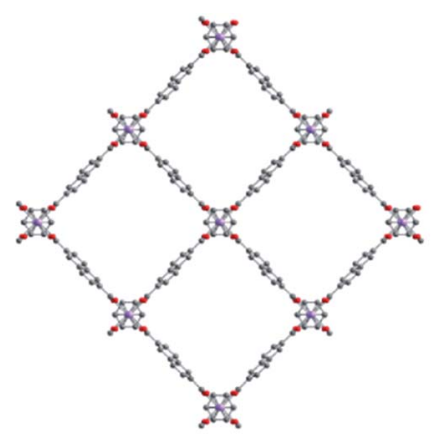

b)

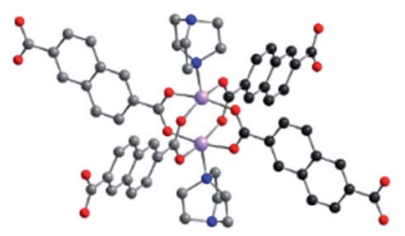

c)

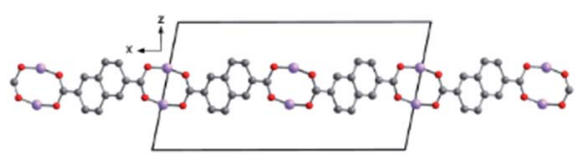

Fig. 4 (a) Crystal structure of $1 a \_o p$ along $c$; (b) the coordination environment of the paddle wheel; (c) view on the layer along $b . C-$ in grey/black, $\mathrm{O}$ - in red, $\mathrm{N}$ - in blue, $\mathrm{Ni}$ - in green, $\mathrm{Co}$ - in purple. Hydrogen atoms are omitted. disorder in the structure. This increases parameters uncertainties in the overall structural model. Resulting unit cell parameters, and as a consequence, unit cell volumes are comparable for 1a_cp and $\mathbf{1 b} \_c p$ structures.

The most noticeable difference between these two structures is the distortion of the paddle wheel units. In the case of $\mathbf{1} \mathbf{b} \_c p$, there is very strong distortion from the square-pyramidal coordination geometry of $\mathrm{Ni}(\mathrm{O}-\mathrm{Ni}-\mathrm{O})$, angles are between $61^{\circ}$ and $110^{\circ}$ (Fig. 3e and $\mathrm{f}$ ) and bending of the carboxylate hinges is expected to be the main origin of the "gate opening" mechanism.

In the case of 1a_cp, the coordination geometry of the Co cluster undergoes only minor changes during the closing and seems to have only minor contribution to the phase transition mechanism. The corresponding $\mathrm{O}-\mathrm{Co}-\mathrm{O}$ angles are between $91^{\circ}$ and $97^{\circ}$ but the $\mathrm{Co}_{2}(\mathrm{COO})_{4}$ unit shows strong shear deformation, altering the angle between the $\mathrm{N} \cdots \mathrm{N}$ (dabco) and $\mathrm{M} \cdots \mathrm{M}$ (paddle wheel) axes significantly (Fig. 3b, d, S16 and S17, ESI $\dagger$ ). The corresponding $\mathbf{M}-\mathbf{M}-\mathbf{N}$ angle in $\mathbf{1 b} \_c p$ structure is $153.9^{\circ}$, whereas in 1a_cp it amounts to $141.5^{\circ}$.

\section{Spectroscopic investigations}

Solid state UV-vis spectroscopy. The huge changes in the coordination geometry during the solvent removal of both structures and the associated visual colour change is reflected in the solid-state UV-vis spectra (Fig. 5a).

In the op phases, a square-pyramidal coordination environment with $C_{4 \mathrm{v}}$ symmetry of each metal atom is assumed. For 1a_op two main signals at 22400 and $17000 \mathrm{~cm}^{-1}$ and two shoulders at 18400 and $14000 \mathrm{~cm}^{-1}$ are observed, which is in good agreement with other reported five-coordinated cobalt complexes.92 1b_op shows only two bands at 24600 and $14700 \mathrm{~cm}^{-1}$ and a small shoulder at $12900 \mathrm{~cm}^{-1}$. These maxima can be assigned to $\mathrm{d} \rightarrow \mathrm{d}^{*}$ transitions $\left({ }^{3} \mathrm{~T}_{1} \rightarrow{ }^{3} \mathrm{~T}_{2}\right)$ of nickel in a square pyramidal or high-spin distorted tetrahedral coordination. ${ }^{93}$ More interestingly, the maxima of 1a_cp show a blue shift with respect to 1a_op while the maxima of 1a_cp show a red shift with respect to $\mathbf{1 b} \_o p$ (Fig. 5a). These observations are linked to the crystallographic data. In 1a_cp the Co $\cdots$ Co distance is shorter compared to that of 1a_op. In contrast, in $\mathbf{1 b} \_c p$ the $\mathrm{Ni} \cdots \mathrm{Ni}$ distance is significantly longer, compared to 1b_op. Furthermore, the higher distortion of the coordination environment in $\mathbf{1} \mathbf{b} \_c p$ is reflected in the bigger change of the respective $\mathrm{M}-\mathrm{N}$ and $\mathrm{M}-\mathrm{O}$ bond lengths (Table 1, Fig. 3e and f).

DFT calculations showed that both HOMO and LUMO are raised in energy upon transition from op to $c p$ form and this is the case for both Co and Ni. However, in the case of $\mathrm{Ni}$, the HOMO is raised significantly more strongly than the LUMO (by approx. $2500 \mathrm{~cm}^{-1}$ ). This results in a smaller HOMO-LUMO gap and is therefore consistent with the redshift in the UV-vis. For $\mathrm{Co}$, the effect is the same according to the conducted calculations, although it is less pronounced (HOMO-LUMO gaps decreases by approx. $1000 \mathrm{~cm}^{-1}$, so a weaker redshift would be expected in some disagreement with the experimental observations). 
Table 1 Characteristic geometric parameters in $M_{2}(b d c)_{2}$ dabco and DUT-8(M) $(M=N i, C o)$ observed by crystallographic investigations

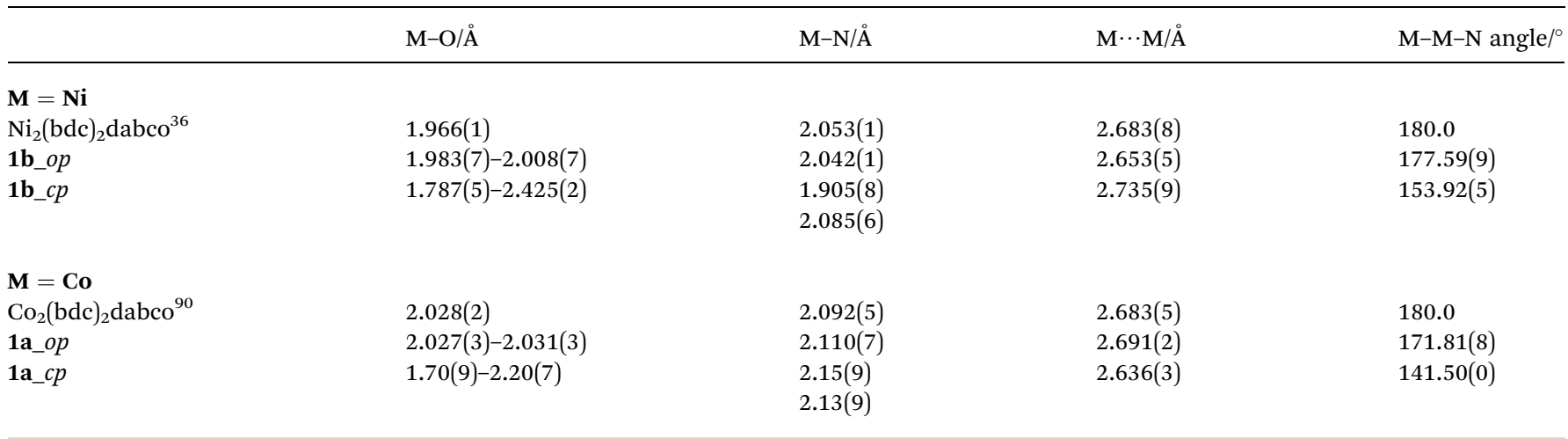

Infrared spectroscopy. From previous studies of DUT-8(Ni) it is known, that the IR spectra of the macro-sized and submicronsized particles differ in some characteristic signals. ${ }^{\mathbf{4 1}}$ The same observation was made for DUT-8(Co) (Fig. S18, ESI $\dagger$ ). The vibrations of the carboxylate $\left(\mathrm{CO}_{2}{ }^{-}\right)$in the recorded range are not significantly influenced by transition metal nature, i.e. the asymmetric stretching vibration is at $1626 \mathrm{~cm}^{-1}$ for both compound in the op phase and at $1602 \mathrm{~cm}^{-1}$ in the $c p$ phase, the symmetric stretching at $1438 \mathrm{~cm}^{-1}$ for both compound in the op phase and $1468 \mathrm{~cm}^{-1}$ in the $c p$ phase, as well as the scissor deformation at $816 \mathrm{~cm}^{-1}$ for $\mathrm{Ni}$ in the $c p$ and $o p$ phases and at $811 \mathrm{~cm}^{-1}$ for Co in the op and $c p$ phases, respectively. ${ }^{94}$ Scissor deformations seems to be more affected by the metal type, then the other. The same is true for the vibration originating from the dabco molecule: asymmetric vibrations are not affected by metal art and slightly affected by coordination geometry change (1060 $\mathrm{cm}^{-1}$ for op phases and $1056 \mathrm{~cm}^{-1}$ for $c p$ phases). But, the symmetric stretching vibrations of $\mathrm{NC}_{3}$ are sensitive and the position of the band differs for Co and Ni compounds in the op phase (Co: $784 \mathrm{~cm}^{-1}, \mathrm{Ni}: 789 \mathrm{~cm}^{-1}$ ), as well as in the $c p$ phase (Co: $776 \mathrm{~cm}^{-1}$, Ni: $769 \mathrm{~cm}^{-1}$, Fig. 5c, Table 2).

Clear differentiation between the op state (material filled with DMF) and the $c p$ state of the macro-sized crystals is possible mainly due to the intense band at $1667 \mathrm{~cm}^{-1}$, which is attributed to the DMF in the pores.

Raman spectroscopy. As shown for DUT-8(Ni), Raman spectroscopy can be used to characterize the open pore phase and the close pore phase by the bands related to phonon modes at $23 \mathrm{~cm}^{-1}$ and $60 \mathrm{~cm}^{-1}$ in the Raman spectra, respectively. ${ }^{96}$ For DUT-8(Co), the corresponding band for open pore phase (1a_op) appears at similar position $\left(24 \mathrm{~cm}^{-1}\right)$, whereas the characteristic lattice vibration band of the closed pore phase $\left(\mathbf{1 a} \_c p\right)$ is shifted to lower frequencies $\left(46 \mathrm{~cm}^{-1}\right)$ in comparison to the 1b_cp, pointing to the subtle differences in the corresponding structures (Fig. 5c, full spectra Fig. S19, ESI†). Since the lowwavenumber range in Raman corresponds to the lattice vibrations, the decrease in frequency for the closed phase from $59 \mathrm{~cm}^{-1}$ for DUT-8(Ni) to $46 \mathrm{~cm}^{-1}$ for DUT-8(Co) indicates that the force constants for DUT-8(Co) framework must be lower with respect to DUT-8(Ni), indicating a softer structure. This may indicate a higher elasticity of the DUT-8(Co) structure or some differences in the pore size of $c p$ phases. The coincidence of some lines of the spectra, corresponding to the vibrations of individual structural elements, reflects the isostructurality of the compounds.

Brillouin spectroscopy. Since no significant differences (within the experimental uncertainness) were found in the low frequency region of the Raman spectra for the op phases of DUT-8(Co) and DUT-8(Ni), the as made compounds were further investigated by Brillouin scattering (Fig. 5d). This provides the possibility to measure the lowest frequencies, including acoustic modes. Moreover, Brillouin spectroscopy allows determination of macroscopic physical parameters, in the first turn sound velocities and elastic moduli, since the speed of sound is directly proportional to the elastic moduli.

The measured Brillouin scattering spectrum for the DUT8 (Ni) op phase at $295 \mathrm{~K}$ (Fig. 5, green line), contains four lines, corresponding to longitudinal acoustic modes. From the fitting, the frequency positions of the scattering lines, $\nu_{\mathrm{B} 1}=8.2$ $\mathrm{GHz}$ and $\nu_{\mathrm{B} 2}=20.0 \mathrm{GHz}$, are obtained. Over time, the peaks at $8.23 \mathrm{GHz}$ disappeared, presumably to due to the slow evaporation of solvent (DMF). The spectrum of the op phase of DUT$8(\mathrm{Co})$ (Fig. 6, blue line) has two sets of broad symmetric lines around $7 \mathrm{GHz}$ and $14 \mathrm{GHz}$. The sharp peak on the right side of the spectrum at $6 \mathrm{GHz}$ corresponds to the laser line. The lines at $7 \mathrm{GHz}$ might correspond to the solvent.

So, there is a significant difference of $6 \mathrm{GHz}$ between the Brillouin line positions in the spectra of DUT-8(Ni) and DUT$8(\mathrm{Co})$ from $20 \mathrm{GHz}\left(0.67 \mathrm{~cm}^{-1}\right)$ to $14 \mathrm{GHz}\left(0.47 \mathrm{~cm}^{-1}\right)$, respectively.

According to Brillouin ${ }^{97}$ the frequency position of the Brillouin line $\left(\nu_{\mathrm{Br}}\right)$ obtained in the backscattering geometry, is proportional to the sound velocity $(u)$ :

$$
\nu_{\mathrm{Br}}=\frac{2 n u}{\lambda}
$$

where $n$ is the refractive index of the scattering medium and $\lambda$ is the excitation wavelength. Therefore, the difference in Brillouin line positions for those samples can be explained by a difference in sound velocities of the samples (or/and the differences in refractive indices). There are no experimental data for the refractive indexes in those samples, but since the Co and $\mathrm{Ni}$ 

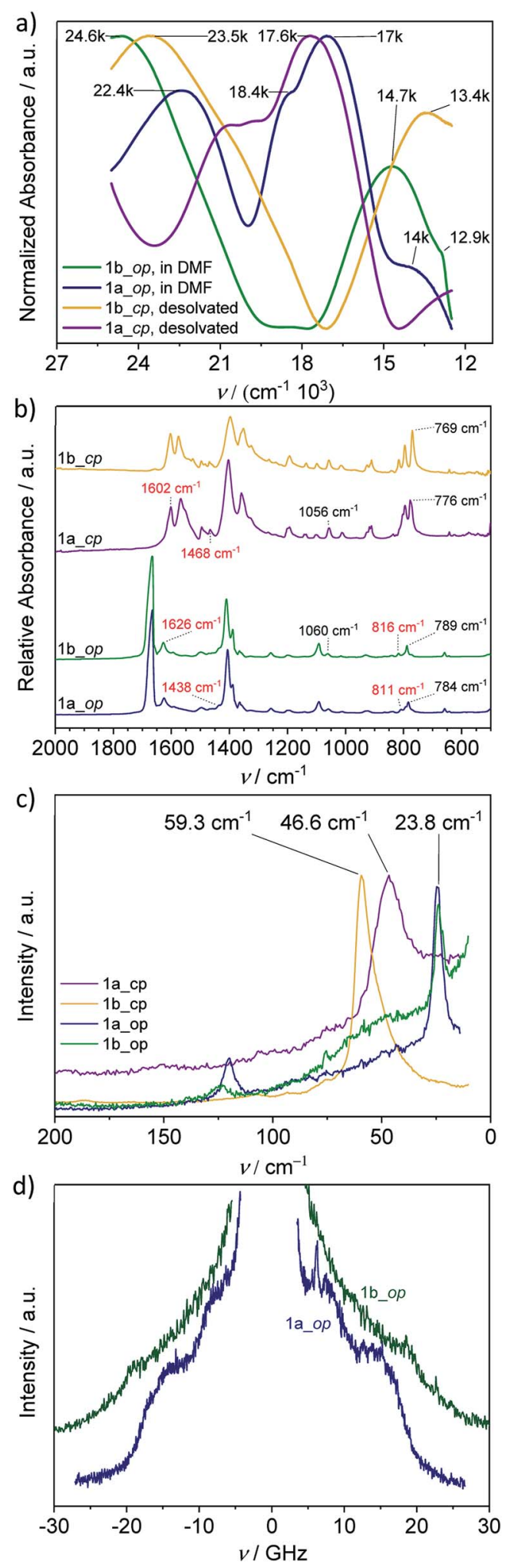

Fig. 5 (a) Solid state UV-vis spectra of 1a_op (blue), 1b_op (green), $1 a \_c p$ (purple) and 1b_cp (yellow); (b) FT-IR spectra of 1a_op (blue), $1 b \_o p$ (green), 1a_cp (violet) and 1b_cp (yellow). Presented wavenumbers attributed to vibrations of carboxylate (red) and dabco (black); (c) The low frequency Raman spectra of 1a_op (blue), 1b_op (green), 1a_cp (purple) and 1b_cp (yellow); (d) Brillouin spectra of $1 \mathrm{a} \_$op (green) and 1b_op (blue).
Table 2 Wavenumbers of expected and observed vibrations

\begin{tabular}{|c|c|c|c|}
\hline Vibration & $\nu$ expected $/ \mathrm{cm}^{-1}$ & $\begin{array}{l}\nu \text { observed } \\
\text { in 1a_cp } / \mathrm{cm}^{-1}\end{array}$ & $\begin{array}{l}\nu \text { observed } \\
\text { in } \mathbf{1} \mathbf{b} \_c p / \mathrm{cm}^{-1}\end{array}$ \\
\hline$\nu_{\mathrm{as}} \mathrm{CO}_{2}^{-}$ & 1695-1540 & 1602 & 1602 \\
\hline$\nu_{\mathrm{S}} \mathrm{CO}_{2}^{-}$ & 1440-1335 & 1468 & 1468 \\
\hline$\delta_{\text {oop }} \mathrm{OH} \cdots \mathrm{O}$ & $860-615$ & 811 & 816 \\
\hline$\nu_{\text {as }} \mathrm{NC}_{3}$ & 1061 (ref. 95) & 1056 & 1056 \\
\hline$\nu_{\mathrm{S}} \mathrm{NC}_{3}$ & 783 (ref. 95) & 776 & 769 \\
\hline
\end{tabular}

based compounds are isomorphous, we assume similar refractive indexes, as it was demonstrated for IRMOF-10 compounds containing different metals. ${ }^{98}$ Thus, one can conclude that the DUT-8(Ni) has a higher sound velocity than DUT-8(Co), suggesting that the op phase of the Co based compound is also softer than the Ni analogue.

\section{Magnetic measurements}

To investigate the magnetic properties of the materials and to shed light on the spin states of the $\mathrm{Ni}(\mathrm{II})$ and $\mathrm{Co}$ (II) ions, temperature dependent static magnetisation $(M)$ measurements were performed on all samples. The effective magnetic moment $\mu_{\text {eff }}$ per metal ion obtained from the static magnetic susceptibility $\chi=M / H$ as $\mu_{\text {eff }}^{2}=3 \chi k_{\mathrm{B}} T / n \mu_{0} \mu_{\mathrm{B}}^{2}$ is presented in Fig. $6 \mathrm{a}$ and $\mathrm{b}$, for DUT-8(Ni) and DUT-8(Co), here $H$ is the static magnetic field, $k_{\mathrm{B}}$ - the Boltzmann constant, $T$ - temperature, $n$ - number of metal ions in the unit cell, $\mu_{0}$ - vacuum permeability, $\mu_{\mathrm{B}}-$ Bohr magneton. At low temperatures $(T<100 \mathrm{~K})$, $\mathbf{1 b} \_o p$ has a very low value of $\mu_{\text {eff }}$ clearly suggesting a nonmagnetic ground state of the studied compound (Fig. 6a). At $T$ $>100 \mathrm{~K} \mu_{\text {eff }}$ increases indicating thermally activated transition to a magnetic state. However, up to $T=300 \mathrm{~K}$, the absolute value of $\mu_{\text {eff }}$ remains significantly lower than $\mu_{\text {eff }}=g\left(S^{2}+S\right)^{1 / 2}=3.1 \mu_{\mathrm{B}}$ expected for the $\mathrm{Ni}$ (II) with $S=1$ and $g=2.2$ (typical $g$-factor for $\mathrm{Ni}(\mathrm{II})$ ions), which means that the transition process is not completed. The magnetic behaviour of compounds $\mathbf{1} \mathbf{b}_{-} c p$ and 2b_op differs significantly from that of 1a_op (Fig. 6). Here, already at lowest temperature $(T=1.8 \mathrm{~K})$, both compounds show finite $\mu_{\text {eff }}$ meaning that a certain fraction of the sample is in a magnetic state. For $\mathbf{1 b} \_c p, \mu_{\text {eff }}$ saturates at room temperature $(T=300 \mathrm{~K})$ reaching a value of $3.0 \mu_{\mathrm{B}}$, which is very close to $\mu_{\text {eff }}=3.1 \mu_{\mathrm{B}}$, suggesting that practically the whole sample is in a magnetic state. $\mathbf{1 b} \_o p$ displays an intermediate situation: $\mu_{\text {eff }}$ increases continuously with increasing the temperature, however even at $T=300 \mathrm{~K}$ its value is significantly smaller than $3.1 \mu_{\mathrm{B}}$. A behaviour similar to that observed for $\mathbf{1} \mathbf{b} \_o p$ was also observed for complexes containing dimeric paddle wheel units. ${ }^{99,100}$ The most common interpretation of this behaviour is an antiferromagnetic (AFM) coupling between the Ni ions in the paddle wheel dimer, which leads to the spin-singlet nonmagnetic ground state, and thermal activation of the magnetic states with the non-zero total spin at higher temperatures. The temperature dependence of the magnetic susceptibility in this case can be described using the Van Vleck equation 
a)

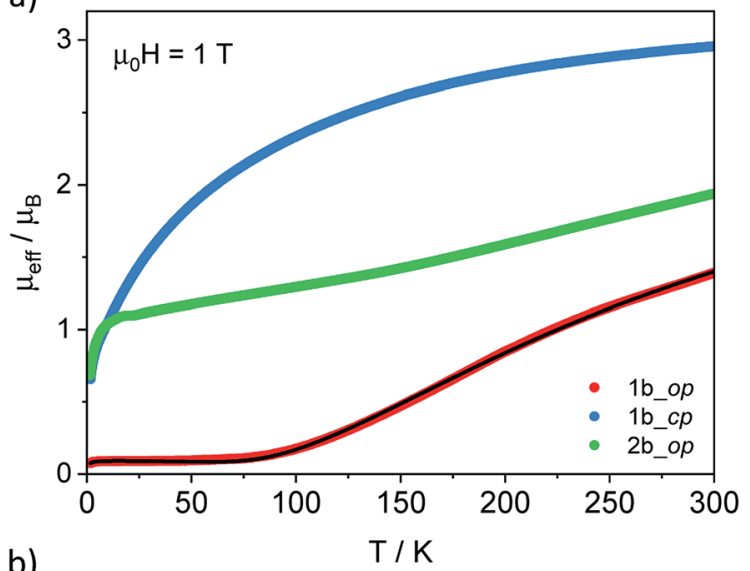

b)

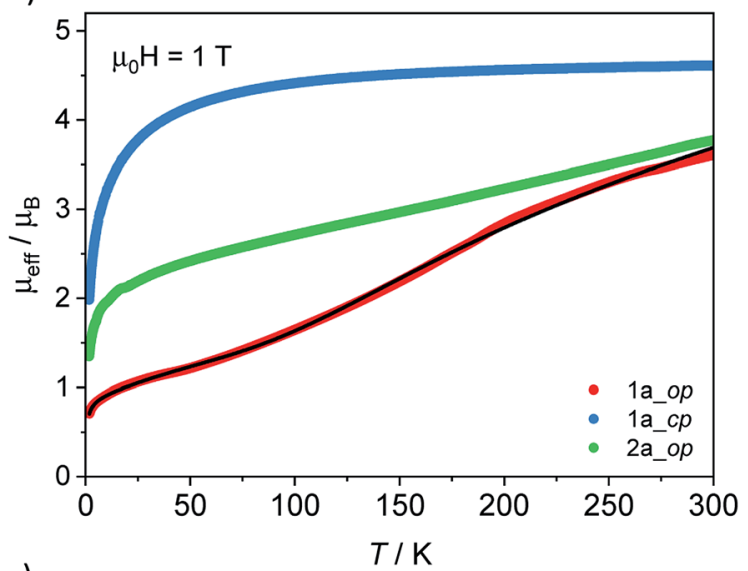

c)

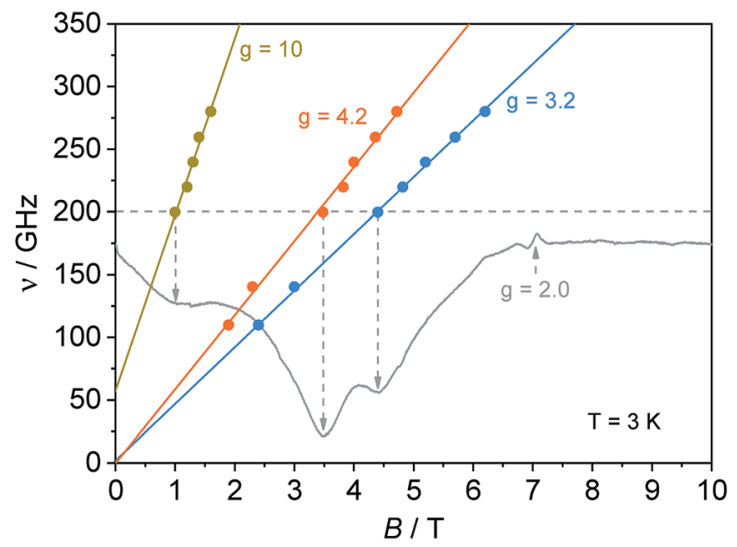

Fig. 6 Temperature dependence of the effective magnetic moment $\mu_{\text {eff }}(T)$ obtained from the static magnetization measurements in the magnetic field of $1 \mathrm{~T}$. (a) DUT-8(Ni); black solid line represents the fit using eqn (2) with $S=1, J=-348 \mathrm{~K}, g=2.9, P=0.001, \Theta=-1 \mathrm{~K}, \chi_{0}=$ $1 \times 10^{-5} \mathrm{emu} \mathrm{Oe}^{-1} \mathrm{~mol}^{-1}$. (b) DUT-8(Co); black solid line represents the fit using eqn (2) with $S=3 / 2, J=256 \mathrm{~K}, g=4.5, P=0.019, \Theta=-1$ $\mathrm{K}, \chi_{0}=4 \times 10^{-3} \mathrm{emu} \mathrm{Oe}^{-1} \mathrm{~mol}^{-1}$. (c) HF-ESR spectrum of 1a_cp measured at the excitation frequency of $200 \mathrm{GHz}$ at $T=3 \mathrm{~K}$ and the frequency $\nu$ vs. resonance field $\mu_{0} H_{\text {res }}$ diagram obtained from the measurements in the frequency range between 100 and $280 \mathrm{GHz}$. Symbols correspond to the resonance fields $H_{\text {res }}^{\mathrm{i}}$ of the individual peaks of the spectrum measured at a fixed $\nu$. Solid lines represents fits of the $H_{\text {res }}^{i}(\nu)$ dependence which slope is determined by the $g$-factor. The value of $g$ for different peaks obtained from the corresponding slopes are indicated in the figure. and an additional term which takes into account a contribution from paramagnetic $\mathrm{Ni}(\mathrm{II})$ spins not coupled in dimers:

$$
\begin{aligned}
\chi= & (1-P) \frac{N_{\mathrm{A}} \mu_{\mathrm{B}}{ }^{2} g^{2}}{3 k_{\mathrm{B}} T} \frac{\sum_{S^{\prime}} S^{\prime}\left(S^{\prime}+1\right)\left(2 S^{\prime}+1\right) \exp \left(\frac{J S^{\prime}\left(S^{\prime}+1\right)}{2 T}\right)}{\sum_{S^{\prime}}\left(2 S^{\prime}+1\right) \exp \left(\frac{J S^{\prime}\left(S^{\prime}+1\right)}{2 T}\right)} \\
& +P \frac{N_{\mathrm{A}} \mu_{\mathrm{B}}{ }^{2} g^{2} S(S+1)}{3 k_{\mathrm{B}}(T-\Theta)}+\chi_{0}
\end{aligned}
$$

here $N_{\mathrm{A}}$ is the Avogadro constant, $S^{\prime}$ is the total spin of the Nidimer which takes the values $S^{\prime}=0,1$, and 2 for the ground state and the two excited spin states of the dimer at energies $J$ and $2 J$, respectively, $J$ is the exchange integral, $P$ is the fraction of not dimerized paramagnetic ions, $\Theta$ is their Curie-Weiss temperature, and $\chi_{0}$ is the temperature independent contribution to the magnetic susceptibility. The best fit of this equation to the experimental data of $\mathbf{1 b}$ _as made, presented in Fig. $6 \mathbf{a}$ as black solid line, yields the AFM coupling constant $J=-348 \mathrm{~K}$ $\left(242 \mathrm{~cm}^{-1}\right)$ between the $\mathrm{Ni}$ spins in the paddle wheel dimer, which is comparable to the values previously observed for complexes with similar structure. ${ }^{\mathbf{1 0 0 , 1 0 1}}$ The fit also reveals $P=$ 0.001 and $\Theta=-1 \mathrm{~K}$, which suggest the presence of $0.1 \%$ of practically non-interacting paramagnetic $\operatorname{Ni}($ II $)(S=1)$ ions in the sample. It should be noted, despite the overall good fit of the curve, the $g$-factor $(g=2.9)$ is larger than $g=2.2$, usually expected for $\mathrm{Ni}(\mathrm{II})$ ions. In contrast to $\mathbf{1 b} \_o p$, the results obtained for $\mathbf{2} \mathbf{b} \_o p$ and $\mathbf{1 b} \_c p$ compounds, cannot be analysed in a similar manner using eqn (2). This is due to the finite magnetic moment at low temperatures and the absence of a clear activation behaviour. The continuous increase of the magnetic moment of the $\mathbf{2} \mathbf{b} \_o p$ sample, in the whole temperature range, can be tentatively interpreted assuming a very broad distribution of $J$ values for the Ni-dimer complexes, which would then smear out the activated behaviour described by eqn (2). In $\mathbf{1} \mathbf{b} \_c p, \mu_{\text {eff }}$ almost saturates at $T=300 \mathrm{~K}$ indicating that the average AFM coupling, $J$, between the Ni spins is significantly smaller than in the other two compounds (estimated below $100 \mathrm{~K})$.

DUT-8(Co) compounds demonstrate qualitatively similar behaviour, see Fig. 6b. For 1a_op, at low temperatures the small value of $\mu_{\text {eff }}$ corresponds to the uncoupled paramagnetic $\mathrm{Co}(\mathrm{II})$ ions with $S=3 / 2(4.5 \%$ according to the fit using eqn (2)) and a major part of the sample in a non-magnetic ground state, due to the antiferromagnetic interaction in the paddle wheel dimer leading to the total spin of the Co-dimer $\left(S^{\prime}=0\right)$.

Upon increasing the temperature, we observe thermal activation of the magnetic moment due to the population of the excited states of the dimers with non-zero total spin, $S^{\prime}=1,2$, and 3. The analysis of this data using eqn (2) yields the AFM $J=$ $-256 \mathrm{~K}\left(178 \mathrm{~cm}^{-1}\right)$. Similar to $2 \mathbf{b} \_o p$, in $2 \mathbf{a} \_o p$, we observe a continuous increase of $\mu_{\text {eff }}$ with increasing the temperature, indicating a broad distribution of the $J$ values in the sample. For 1a_cp, $\mu_{\text {eff }}$ increases with temperature and saturates already at $T$ $>100 \mathrm{~K}$, which suggests a small coupling between the Co spins $(J<50 \mathrm{~K})$. 
High-field/high-frequency electron spin resonance (HF-ESR) measurements were performed on the 1a_ $c p$ compound, in order to determine the $g$-factor of $\mathrm{Co}(\mathrm{II})$ ions. A representative ESR spectrum together with the corresponding frequency $v s$. resonance magnetic field diagram is presented in Fig. 6c. The spectrum consists of several overlapping and very broad absorption lines with different $g$-factors, which is likely caused by some inhomogeneity of the sample. The observed $g$-values around 4.2 and 3.2 are typical for the perpendicular $g$-factor $\left(g_{\perp}\right)$ of Co(II) ions in metal-organic compounds. ${ }^{102,103}$ Signal from the parallel orientation $\left(g_{\|}\right.$, typical values are around 1.9-2.0) is not visible in the spectrum, probably due to the smaller intensity. Furthermore, there may be a possible preferential orientation of the powder particles in the sample, which boosts the contribution to the total spectrum for particles with local symmetry axis of the Co-ligand clusters oriented perpendicular to the magnetic field. Thus the expected effective (powder averaged) $g$ factor for $1 a_{-} c p$ can be estimated as $g_{\text {av. }} \approx 3.1$, which is smaller than $g=4.5$ obtained for 1a_op from fitting of the magnetization data. The left-most line in the ESR spectrum in Fig. 6c characterized with a large $g$-factor $g=10$ could be attributed to the so-called "forbidden" ESR transition within the multiplet of the spin levels of $\mathrm{Co}$ (II) ions with $S=3 / 2$ featuring by its virtue a large $g$-factor. Note that the "forbidden" transition is possible only in the spin multiplet with $S>1 / 2$ and therefore the presence of such a line in the ESR spectrum of the 1a_cp compound provides additional evidence of the high-spin state of the $\mathrm{Co}$ (II) ions in this material. Finally, the low-intensity narrow ESR line with $g=2.0$ in the spectrum most probably originates from a small amount of paramagnetic impurities in the sample.

To summarize the analysis of magnetic data, both $\mathbf{1 b} \_o p$ and 1a_op exhibit a spin-singlet non-magnetic ground state due to the antiferromagnetic interaction of the magnetic ions in the paddle wheel dimer and a thermal activation of the magnetic moment due to occupation of excited spin states of the dimer at higher temperatures. Both $\mathbf{2} \mathbf{b} \_o p$ and $\mathbf{2 a} \_o p$ compounds can be characterized by a very wide distribution of the exchange integral $J$ probably due to the structural inhomogeneity in the sample and increased concentration of defects. ${ }^{42}$ On the other hand, both 1) $\mathbf{b} \_c p$ and 1a_cp compounds show a rather small average coupling in comparison to the aforementioned samples. This could be possibly related to significant differences in the structure between the submicron-sized (2) and macro-sized (1) compounds. Indeed, the strength of the superexchange coupling $J$ is strongly influenced by the geometry of the bonds connecting the magnetic ions. ${ }^{103}$

\section{Density functional theory}

Spin related cluster optimisation. To assess the intrinsic impact of a different metal, $\mathrm{Ni}$ and $\mathrm{Co}$, on the structure and flexibility of the paddle wheel motif, as well as impact of the spin state, density functional theory (DFT) simulations were conducted on a simple molecular analogue of the paddle wheel cluster comprised of ammonia and formate ligands (Fig. S20, ESI†).

Since the spin configuration is known to significantly impact the geometry of the Co complexes ${ }^{104}$ and the magnetic measurements demonstrated possible variation of spin states in DUT-8 compounds, depending on the temperature, network geometry and solvation state, initially, geometric structures of the paddle wheel clusters were optimised in both low-spin and high-spin configurations. The low-spin state corresponds to an antiferromagnetic singlet arrangement for both $\mathrm{Ni}$ and $\mathrm{Co}$ paddle wheel structures while the high-spin state corresponds to a quintet state (multiplicity $M=5$ ) for Ni and triplet $(M=3)$ and septet $(M=7)$ states for Co, alternatively 4,2 and 6 unpaired electrons per paddle wheel, respectively. Notably, optimised structures of these different spin states are significantly different (Fig. S21, ESI $\dagger$ ). High-spin configurations produce substantially deformed geometries with $\mathrm{N}-\mathrm{M}-\mathrm{M}$ angles (where $\mathrm{M}=\mathrm{Co}$ or $\mathrm{Ni}$ ) of $<165^{\circ}$ which interestingly is a similar distortion to that observed in DUT-8 closed pore structures. Critical parameters of the optimised structures are detailed in Table 3.

Geometries of the high-spin configurations of the paddle wheel cluster expectedly demonstrate longer $\mathbf{M} \cdots \mathrm{M}$ distances compared to the low-spin systems due to the unpaired electrons populating non-bonding orbitals, similar to that observed for the $\mathrm{Cu}$ paddle wheel. ${ }^{105}$ When compared to the geometry of the clusters observed in DUT-8_op single crystal structures, the Ni paddle wheel shows good agreement. However, the Co $\cdots$ Co distance obtained from the crystal structure $(2.691 \AA)$ is not reflected by a single predicted spin state. The Co $\cdots$ Co distance observed in the experimental crystal structures may suggest a combination of spin states within the DUT-8(Co) framework.

To explore the intrinsic flexibility of the paddle wheel clusters, we considered constrained geometric optimisation of the representative molecules. For these simulations the $\mathrm{N}-\mathrm{M}-\mathrm{M}$ and $\mathrm{M}-\mathrm{M}-\mathrm{N}$ angles were fixed and subsequently scanned for angles between $175^{\circ}$ and $150^{\circ}$. Both low and high-spin configurations (total spin in the $\mathrm{M}_{2}$ paddle wheel) were considered for DUT-8(Co) (Fig. S22, ESI†) and DUT-8(Ni) (Fig. S23, ESI†). The geometry scans reveal the flexibility inherent to the paddle wheel cluster and the profound effect of different spin configurations. A high spin state of the Co $\cdots$ Co cluster $(M=7)$ has a completely different minimum to the other considered spin states where the minimum structures display an extremely similar geometry to the distorted paddle wheel structures observed experimentally in the $c p$ phase of DUT-8. Notably, an energy penalty of $>10 \mathrm{~kJ} \mathrm{~mol}^{-1}\left(\mathrm{Co}_{2}\right)$ is required to straighten the paddle wheel to a geometry consistent with the structure of the op phase of DUT-8(Co) (Fig. 7a). These DFT simulations described here illustrate the powerful effect spin states have on the geometry of paddle wheel clusters. The Co system is

Table 3 Critical bond lengths and angles of the optimised paddle wheel structures. Multiplicity $(M)$ is given in the parentheses $(M=2 S+$ 1 , where $S$ is the total spin angular momentum)

\begin{tabular}{llcccl}
\hline & $\mathrm{Ni} \cdots \mathrm{Ni}(\mathbf{1})$ & $\mathrm{Ni} \cdots \mathrm{Ni}(\mathbf{5})$ & $\mathrm{Co} \cdots \mathrm{Co}(\mathbf{1})$ & $\mathrm{Co} \cdots \mathrm{Co}(3)$ & $\mathrm{Co} \cdots \mathrm{Co}(7)$ \\
\hline $\mathrm{M} \cdots \mathrm{M} / \AA$ & 2.65 & 2.70 & 2.44 & 2.56 & 2.82 \\
$\mathrm{~N}-\mathrm{M}-\mathrm{M} /{ }^{\circ}$ & 180 & 164.9 & 178.2 & 165.3 & 156
\end{tabular}


predicted to have a septet spin state however we note that DFT simulations of relative spin energetics is challenging. ${ }^{106}$

Spin related framework optimisation. Calculations of the periodic structure with GGA+U correction $(U-J=2.2 \mathrm{eV})$ are in line with the high-level calculations performed on cluster models. Namely, the strain in the structure depends on the spin state of the Co ions and decreases from a low to high-spin state (Fig. S24, ESI $\dagger$ ). The periodic calculations also represent well the energy splitting of $90 \mathrm{~kJ} \mathrm{~mol}^{-1}$ between CoCo (1) and CoCo (7) together with the Co $\cdots$ Co distance in the paddle wheel units, which is 2.50 and $2.79 \AA$ in the periodic calculations vs. 2.44 and $2.82 \AA$ in the cluster calculations, respectively.

The energy difference between the most stable state of the periodic $c p$ and $o p$ phase is $-91 \mathrm{~kJ} \mathrm{~mol}^{-1}$ per unit cell $\left(\mathrm{Co}_{2}\right.$ and two 2,6-ndc linkers). The strain ( $E(\mathrm{str}))$ is $84 \mathrm{~kJ} \mathrm{~mol}^{-1}$ per unit cell, which is $18 \mathrm{~kJ} \mathrm{~mol}^{-1}$ smaller in comparison to the corresponding strain in DUT-8(Ni). This result is in line with the Brillouin spectroscopy, as discussed above. The dispersion energy difference $(\Delta E(\operatorname{disp}))$ between $c p$ an op phase is also somewhat smaller in comparison to DUT-8(Ni), but only by $13 \mathrm{~kJ} \mathrm{~mol}^{-1}$ (Tables 4 and $\mathrm{S} 1 \dagger$ ). Thus, overall energetic balance shows slightly higher stability of the $c p$ phase of DUT-8(Co) in comparison to DUT-8(Ni).

Spin related UV-vis spectra simulation. The UV-vis spectra of DUT-8(Ni) were modelled using TDA-TD-DFT on a cluster model assuming an open-shell singlet spin state resulting from two unpaired electrons per metal atom with antiferromagnetic coupling between the two adjacent spin centers. After blueshifting the calculated spectrum by $750 \mathrm{~cm}^{-1}$, very good agreement with the experimental data is achieved (Fig. 7c). All strong absorptions in the visible range can be attributed to $\mathrm{d} \rightarrow$ d transitions. Apart from the bands in the regions of $20000-$ $23000 \mathrm{~cm}^{-1}$ and $13000-5000 \mathrm{~cm}^{-1}$, which have strong $\mathrm{d}_{x z} / \mathrm{d}_{y z}$ $\rightarrow \mathrm{d}_{x y} / \mathrm{d}_{x^{2}-y^{2}}$ and $\mathrm{d}_{x y} \rightarrow \mathrm{d}_{z^{2}}$ character, respectively, TD-DFT predicts a third maximum around $10000 \mathrm{~cm}^{-1}$ with $\mathrm{d}_{x z} / \mathrm{d}_{y z} \rightarrow$ $\mathrm{d}_{z^{2}}$ character. It should be noted, though, that the abovementioned d orbitals interact with the ligand orbitals to split into a large variety of molecular orbitals and each transition can be decomposed into a multitude of contributing orbitals, complicating an intuitive understanding of the transitions. Upon transition from the open pore to the closed pore phase of the MOF, all $\mathrm{d} \rightarrow \mathrm{d}$ transitions are red-shifted, this effect being strongest for the highest-energy transitions. Apart from $\mathrm{d} \rightarrow$ $\mathrm{d}$ transitions, also $\pi \rightarrow \pi$ transitions occur; the lowest ones reach into the blue region of the spectrum, but are covered by the much stronger $\mathrm{d} \rightarrow \mathrm{d}$ transitions.

As a $\mathrm{d}^{7}$ system, $\mathrm{Co}^{2+}$ gives a more complicated electronic structure than $\mathrm{Ni}^{2+}$. For the TD-DFT calculations, both ferromagnetic and antiferromagnetic coupling between both lowspin and high-spin (one and three unpaired electrons per metal center, respectively) were considered. Unfortunately, the higher number of transitions within a narrow spectral range and the errors inherent to state-of-the-art TD-DFT approximations make it difficult to compare the calculated spectra with the experimental data. The spectra calculated for the high-spin open pore form are consistent with the experimental data. Spectra calculated for ferromagnetic and antiferromagnetic
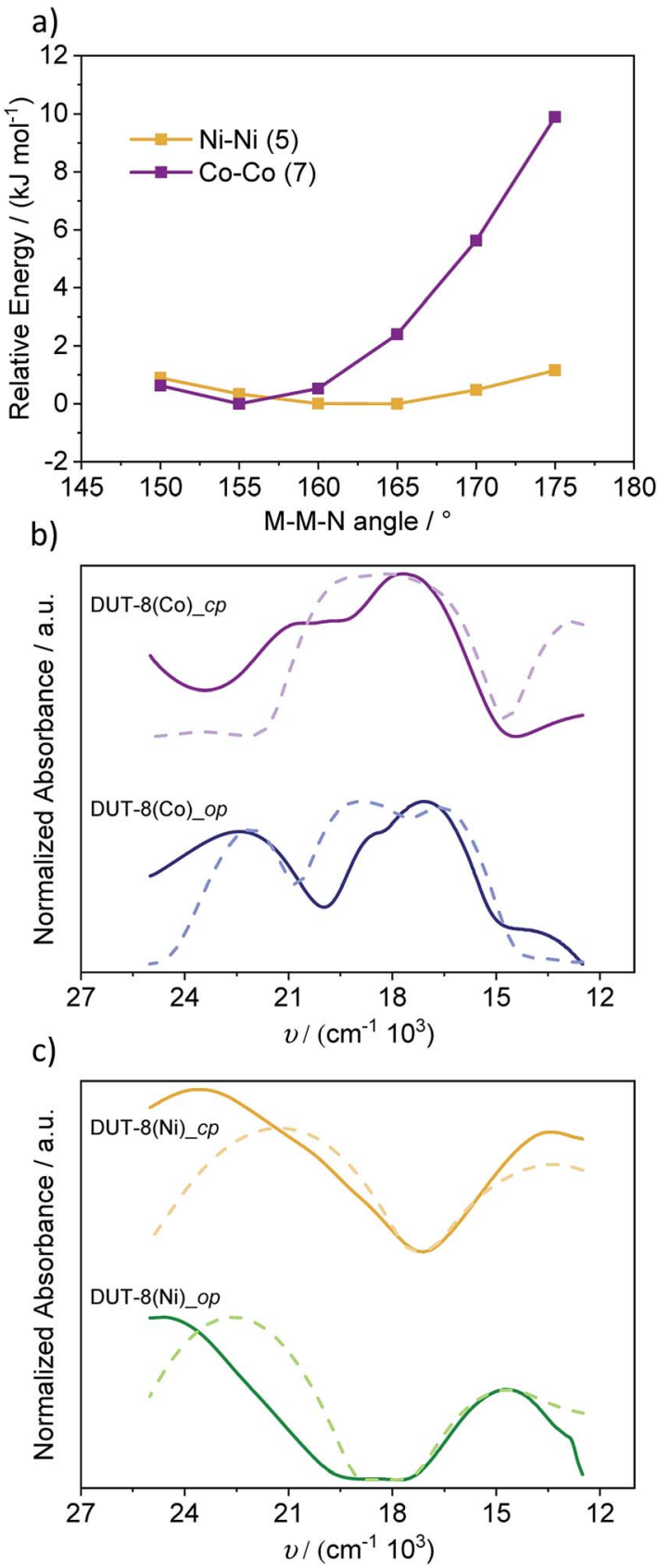

Fig. 7 (a) Comparison of the constrained geometry scans of the $\mathrm{M}-\mathrm{M}-\mathrm{N}$ angles for the representative $\mathrm{Co}$ (violet) and $\mathrm{Ni}$ (yellow) paddle wheel clusters. Simulated UV-vis spectra of (b) DUT-8(Co) and (c) DUT-8(Ni). Solid lines represent the experimental and dotted lines the simulated data. Calculated spectra for both forms of DUT-8(Ni) have been blueshifted by $750 \mathrm{~cm}^{-1}$, for DUT-8(Co) only the closed pore form was blueshifted by $1500 \mathrm{~cm}^{-1}$.

high-spin states are very similar (Fig. S26, ESI $\dagger$ ) and equally plausible on the basis of the UV/vis data alone. However, neither of the spectra calculated for the high-spin state explain the experimental peak at $22400 \mathrm{~cm}^{-1}$. The latter is more in line with the spectra calculated for the low-spin state, which predict peaks at $22100 \mathrm{~cm}^{-1}$ and at $20300 \mathrm{~cm}^{-1}$ for ferromagnetic and antiferromagnetic coupling, respectively. Comparing the 
Table 4 The energy differences calculated between the most stable $o p$ and $c p$ phases for DUT- 8 compounds (for more detailed information see Table S1) ${ }^{a}$

\begin{tabular}{llll}
\hline & $\begin{array}{l}\Delta E(c p-o p) / \mathrm{kJ} \\
\mathrm{mol}^{-1}\end{array}$ & $\begin{array}{l}\Delta E(\mathrm{disp})(c p-o p) / \mathrm{kJ} \\
\mathrm{mol}^{-1}\end{array}$ & $\begin{array}{l}E(\mathrm{str})(c p-o p) / \mathrm{kJ} \\
\mathrm{mol}^{-1}\end{array}$ \\
\hline DUT-8(Co) & -91 & -175 & 84 \\
DUT-8(Ni) & -86 & -188 & 102
\end{tabular}

${ }^{a} 1 \mathrm{~mol}$ in the simulations contains $\mathrm{M}_{2}$ paddle wheel unit, two 2,6-ndc linkers, and 1 dabco molecule.

wavenumbers, the ferromagnetic state may seem more plausible, but since the spectra corresponding to different spin states likely exhibit different wavenumber shifts, the antiferromagnetic low-spin state is also possible. In conclusion, the experimental spectrum of the open-pore DUT- $8(\mathrm{Co})$ is most consistent with a majority of high-spin Co centers and a minority of approximately 5 to $25 \%$ of paddle wheels in a lowspin state. In contrast to that of DUT-8(Ni), the UV-vis spectrum of DUT-8(Co) apparently blue-shifts upon transition to the closed pore form. Assuming the same spin state for open and closed pore form, none of our calculations are able to explain this blue shift and satisfying qualitative agreement between the calculated spectrum of the closed pore form with respect to the experimental spectrum is only achieved after blue-shifting the former by $1500 \mathrm{~cm}^{-1}$ (Fig. S25, ESI $\dagger$ ).

The blue shift could be explained with the calculated spectra only by assuming different spin states for the two phases: the above-mentioned spin state mixture for the open pore phase and an antiferromagnetic low-spin configuration for the closed pore phase. The latter spectrum would also be the best fit for the experimental spectrum. However, the low spin state is unlikely in light of the magnetic measurements. Therefore, despite not being able to give an explanation of the blue shift and a slightly worse fit of the spectrum, the antiferromagnetic high-spin state seems more plausible overall (Fig. 7b).

\section{Discussion}

DUT-8(Ni) as well as DUT-8(Co) show particle size dependent desolvation behaviour. The crystallites smaller than certain critical size do not show closing upon solvent removal and behave as rigid microporous materials in physisorption of gases and vapours. The micrometre-sized crystals of both materials transform to closed pore forms and display pronounced differences in adsorption behaviour. While $\mathrm{Ni}$ based compounds demonstrate a distinct "gate opening" effect for nitrogen at $77 \mathrm{~K}$, carbon dioxide at $195 \mathrm{~K}$ and $n$-butane at $298 \mathrm{~K}$, the Co compound stays in the closed pore form under such adsorption conditions.

Only one adsorptive, among the adsorptives investigated, was able to provoke the structural $c p \rightarrow o p$ transformation of DUT-8(Co), namely DCM $\left(\mathrm{CH}_{2} \mathrm{Cl}_{2}\right)$ at $298 \mathrm{~K}$. Interestingly, exposed to other chlorinated methanes, the Co based framework remains closed, suggesting highly selective adsorption properties, in contrast to the $\mathrm{Ni}$ analogue.
The underlying structural reason for these pronounced differences in switchability and adsorption behaviour lies in subtle differences in the crystal structures of the closed pore phases and deformability of the $\mathrm{Co}_{2}$ paddle wheel unit with roughly $18 \mathrm{~kJ} \mathrm{~mol}^{-1}$ lower strain energy in comparison to $\mathrm{Ni}$. This enhanced deformation upon closing is also reflected in the larger $\mathrm{M}-\mathrm{N}$ bond length and larger $\mathrm{M}-\mathrm{M}-\mathrm{N}$ angle, as well as in the results of low frequency Raman and Brillouin spectroscopy. Both spectroscopic methods point to lower force constants as well as to smaller elastic moduli for DUT-8(Co) framework.

The high barrier for reopening of DUT-8(Co)_cp can be associated to a differences in the spin states of $o p$ and $c p$ phases and a much stepper potential for the $\mathrm{Co}_{2}$-complex in the $c p$ form.

This high energetic barrier causes a highly selective pore opening mechanism resulting in selective recognition of DCM.

The high selectivity for DCM cannot be solely attributed to adsorption thermodynamics, as the adsorption enthalpies for $\mathrm{CCl}_{4}$ are comparable to that of DCM, and adsorption enthalpy of chloroform is even higher. But as the "gate opening" is an activated process, the results suggest an important contribution for the selective pore opening from favourable kinetics of DCM adsorption and the smaller size of the adsorptive facilitating the entry at the openings of the closed channels.

\section{Conclusions}

Investigations of the particle size dependent "gate opening" transitions in DUT-8(Co) reveal striking differences as compared to the Ni analogue. While submicron-sized particles show type Ia isotherms and no switchability, macro-sized samples close during desolvation and cannot be reopened using weakly adsorbing molecules such as $\mathrm{N}_{2}, \mathrm{CO}_{2}$, but even $\mathrm{CHCl}_{3}$ and $\mathrm{CCl}_{4}$ also do not provoke "gate opening". However, an unprecedented high selectivity for "gate opening" induced by $\mathrm{CH}_{2} \mathrm{Cl}_{2}$ and DMF is observed. In depth structural investigations reveal distinct differences in both the coordination geometry of the closed pore state and the tilt of the metal cluster in the respective frameworks. These results were supported by spectroscopic measurements. Furthermore, Raman spectroscopy showed pronounced differences in lattice vibration energetics pointing towards characteristic differences in framework elastic constants. The combined theoretical and experimental approach gives comprehensive insights into the importance of structural building blocks (here the metal centre) versus counteracting morphological characteristics (particle size). This alone is an important step towards rationalization of framework switchability and the development of a predictive theoretical framework for flexible MOFs.

From an application point of view, DUT-8(Co) stands out as a highly selective material for separation, sensing and potentially catalysis, since the material shows this remarkable selective pore opening for $\mathrm{CH}_{2} \mathrm{Cl}_{2}$ vs. $\mathrm{CHCl}_{3}$ and $\mathrm{CCl}_{4}$. Moreover, the changes in magnetism in the course of $o p \rightarrow c p$ transformation are worthwhile to be further investigated in future as they imply interesting coupling of simultaneous magnetic and porosity switching. 


\section{Conflicts of interest}

There are no conflicts to declare.

\section{Acknowledgements}

The authors thank DFG (FOR 2433) for financial support. We thank HZB for the allocation of synchrotron radiation beamtime and financial support. PP, TW and TH used high performance facilities of ZIH Dresden. TW thanks the European Social Funds for Germany for a PhD fellowship.

\section{Notes and references}

1 G. Férey and C. Serre, Chem. Soc. Rev., 2009, 38, 1380.

2 S. Bureekaew, S. Amirjalayer and R. Schmid, J. Mater. Chem., 2012, 22, 10249.

3 J. D. Evans, L. Bocquet and F.-X. Coudert, Chem, 2016, 1, 873.

4 L. Sarkisov, R. L. Martin, M. Haranczyk and B. Smit, J. Am. Chem. Soc., 2014, 136, 2228.

5 S. Krause, V. Bon, I. Senkovska, U. Stoeck, D. Wallacher, D. M. Többens, S. Zander, R. S. Pillai, G. Maurin and F.-X. Coudert, Nature, 2016, 532, 348.

6 E. J. Carrington, C. A. McAnally, A. J. Fletcher, S. P. Thompson, M. Warren and L. Brammer, Nat. Chem., 2017, 9, 882.

7 D. Fairen-Jimenez, S. Moggach, M. Wharmby, P. Wright, S. Parsons and T. Duren, J. Am. Chem. Soc., 2011, 133, 8900.

8 A. Schneemann, P. Vervoorts, I. Hante, M. Tu, S. Wannapaiboon, C. Sternemann, M. Paulus, D. F. Wieland, S. Henke and R. A. Fischer, Chem. Mater., 2018, 30, 1667.

9 S. Kitagawa and M. Kondo, Bull. Chem. Soc. Jpn., 1998, 71, 1739.

10 G. Férey, Chem. Mater., 2001, 13, 3084.

11 J. A. Mason, J. Oktawiec, M. K. Taylor, M. R. Hudson, J. Rodriguez, J. E. Bachman, M. I. Gonzalez, A. Cervellino, A. Guagliardi and C. M. Brown, Nature, 2015, 527, 357.

12 B. Zornoza, A. Martinez-Joaristi, P. Serra-Crespo, C. Tellez, J. Coronas, J. Gascon and F. Kapteijn, Chem. Commun., 2011, 47, 9522.

13 A. Chakraborty, S. Roy, M. Eswaramoorthy and T. K. Maji, J. Mater. Chem. A, 2017, 5, 8423.

14 P. Freund, I. Senkovska and S. Kaskel, ACS Appl. Mater. Interfaces, 2017, 9, 43782.

15 A. Douvali, A. C. Tsipis, S. V. Eliseeva, S. Petoud, G. S. Papaefstathiou, C. D. Malliakas, I. Papadas, G. S. Armatas, I. Margiolaki and M. G. Kanatzidis, Angew. Chem., 2015, 127, 1671.

16 S. Yuan, L. Zou, H. Li, Y. P. Chen, J. Qin, Q. Zhang, W. Lu, M. B. Hall and H. C. Zhou, Angew. Chem., Int. Ed., 2016, 55, 10776.

17 A. Schneemann, V. Bon, I. Schwedler, I. Senkovska, S. Kaskel and R. A. Fischer, Chem. Soc. Rev., 2014, 43, 6062.

18 S. S. Nagarkar, A. V. Desai and S. K. Ghosh, Chem.-Asian J., 2014, 9, 2358.
19 F.-X. Coudert, Chem. Mater., 2015, 27, 1905.

20 Z. Chang, D.-H. Yang, J. Xu, T.-L. Hu and X.-H. Bu, Adv. Mater., 2015, 27, 5432.

21 J.-P. Zhang, H.-L. Zhou, D.-D. Zhou, P.-Q. Liao and X.-M. Chen, Natl. Sci. Rev., 2017, 5, 907.

22 T. D. Bennett, A. K. Cheetham, A. H. Fuchs and F.-X. Coudert, Nat. Chem., 2017, 9, 11.

23 F. Salles, G. Maurin, C. Serre, P. L. Llewellyn, C. Knöfel, H. J. Choi, Y. Filinchuk, L. Oliviero, A. Vimont and J. R. Long, J. Am. Chem. Soc., 2010, 132, 13782.

24 H. J. Choi, M. Dinca and J. R. Long, J. Am. Chem. Soc., 2008, 130, 7848.

25 F. Millange, C. Serre and G. Férey, Chem. Commun., 2002, 822.

26 Y. Jiao, Z. Li, Y. Ma, G. Zhou, S. Wang and G. Lu, AIP Adv., 2017, 7, 085009.

27 F. Millange and R. I. Walton, Isr. J. Chem., 2018, 58, 1019.

28 S. Wannapaiboon, A. Schneemann, I. Hante, M. Tu, K. Epp, A. L. Semrau, C. Sternemann, M. Paulus, S. J. Baxter and G. Kieslich, Nat. Commun., 2019, 10, 346.

29 F. ZareKarizi, M. Joharian and A. Morsali, J. Mater. Chem. A, 2018, 6, 19288.

30 S. Henke, A. Schneemann, A. Wütscher and R. A. Fischer, J. Am. Chem. Soc., 2012, 134, 9464.

31 Z.-H. Xuan, D.-S. Zhang, Z. Chang, T.-L. Hu and X.-H. Bu, Inorg. Chem., 2014, 53, 8985.

32 X.-L. Luo, Z. Yin, M.-H. Zeng and M. Kurmoo, Inorg. Chem. Front., 2016, 3, 1208.

33 K. Seki and W. Mori, J. Phys. Chem. B, 2002, 106, 1380.

34 D. N. Dybtsev, H. Chun and K. Kim, Angew. Chem., 2004, 116, 5143.

35 H. Wang, J. Getzschmann, I. Senkovska and S. Kaskel, Microporous Mesoporous Mater., 2008, 116, 653.

36 P. Maniam and N. Stock, Inorg. Chem., 2011, 50, 5085.

37 N. Klein, H. C. Hoffmann, A. Cadiau, J. Getzschmann, M. R. Lohe, S. Paasch, T. Heydenreich, K. Adil, I. Senkovska and E. Brunner, J. Mater. Chem., 2012, 22, 10303.

38 N. Kavoosi, T. Savchenko, I. Senkovska, M. Maliuta, V. Bon, A. Eychmüller and S. Kaskel, Microporous Mesoporous Mater., 2018, 271, 169.

39 V. Bon, N. Klein, I. Senkovska, A. Heerwig, J. Getzschmann, D. Wallacher, I. Zizak, M. Brzhezinskaya, U. Mueller and S. Kaskel, Phys. Chem. Chem. Phys., 2015, 17, 17471.

40 H. Miura, V. Bon, I. Senkovska, S. Ehrling, S. Watanabe, M. Ohba and S. Kaskel, Dalton Trans., 2017, 46, 14002.

41 N. Kavoosi, V. Bon, I. Senkovska, S. Krause, C. Atzori, F. Bonino, J. Pallmann, S. Paasch, E. Brunner and S. Kaskel, Dalton Trans., 2017, 46, 4685.

42 M. Mendt, F. Gutt, N. Kavoosi, V. Bon, I. Senkovska, S. Kaskel and A. Pöppl, J. Phys. Chem. C, 2016, 120, 14246. 43 U. Mueller, R. Förster, M. Hellmig, F. U. Huschmann, A. Kastner, P. Malecki, S. Pühringer, M. Röwer, K. Sparta, M. Steffien, M. Ühlein, P. Wilk and M. S. Weiss, Eur. Phys. J. Plus, 2015, 130, 141.

44 K. M. Sparta, M. Krug, U. Heinemann, U. Mueller and M. S. Weiss, J. Appl. Crystallogr., 2016, 49, 1085. 
45 A. L. Spek, Acta Crystallogr., Sect. D: Biol. Crystallogr., 2009, 65, 148.

46 SAINT Bruker, Bruker AXS Inc., Madison, Wisconsin, USA, 2012.

47 SADABS Bruker, Bruker AXS Inc., Madison, Wisconsin, USA, 2012.

48 G. M. Sheldrick, Acta Crystallogr., Sect. A: Found. Crystallogr., 2008, 64, 112.

49 D. M. Többens and S. Zander, JLSRF, 2016, 2, 49.

50 V. Bon, I. Senkovska, D. Wallacher, A. Heerwig, N. Klein, I. Zizak, R. Feyerherm, E. Dudzik and S. Kaskel, Microporous Mesoporous Mater., 2014, 188, 190.

51 Materials Studio 5.0, Accelrys Software Inc., San Diego, California, USA, 2009.

52 M. Valiev, E. J. Bylaska, N. Govind, K. Kowalski, T. P. Straatsma, H. J. Van Dam, D. Wang, J. Nieplocha, E. Apra and T. L. Windus, Comput. Phys. Commun., 2010, 181, 1477.

53 C. Adamo and V. Barone, J. Chem. Phys., 1999, 110, 6158.

54 F. Weigend, Phys. Chem. Chem. Phys., 2006, 8, 1057.

55 T. Soda, Y. Kitagawa, T. Onishi, Y. Takano, Y. Shigeta, H. Nagao, Y. Yoshioka and K. Yamaguchi, Chem. Phys. Lett., 2000, 319, 223.

56 M. Krack and M. Parrinello, High performance computing in chemistry, 2004, vol. 25, p. 29.

57 J. VandeVondele, M. Krack, F. Mohamed, M. Parrinello, T. Chassaing and J. Hutter, Comput. Phys. Commun., 2005, $167,103$.

58 N. M. Atherton, Principles of electron spin resonance, Ellis Horwood Limited, 1993.

59 G. Lippert, M. Parriello and J. Hutter, Mol. Phys., 1997, 92, 477.

60 S. Goedecker, M. Teter and J. Hutter, Phys. Rev. B: Condens. Matter Mater. Phys., 1996, 54, 1703.

61 C. Hartwigsen, S. Gœdecker and J. Hutter, Phys. Rev. B: Condens. Matter Mater. Phys., 1998, 58, 3641.

62 M. Krack, Theor. Chem. Acc., 2005, 114, 145.

63 S. Grimme, J. Antony, S. Ehrlich and H. Krieg, J. Chem. Phys., 2010, 132, 154104.

64 F. Neese, Wiley Interdiscip. Rev.: Comput. Mol. Sci., 2012, 2, 73.

65 F. Neese, F. Wennmohs, A. Hansen and U. Becker, Chem. Phys., 2009, 356, 98.

66 M. G. Medvedev, I. S. Bushmarinov, J. Sun, J. P. Perdew and K. A. Lyssenko, Science, 2017, 355, 49.

67 L. Barbour, E. Engel, A. Jouaiti, C. Bezuidenhout and W. Hosseini, Angew. Chem., 2017, 56, 8874.

68 J. Ma, A. P. Kalenak, A. G. Wong-Foy and A. J. Matzger, Angew. Chem., Int. Ed., 2017, 56, 14618.

69 D. Farrusseng, C. Daniel, C. Gaudillere, U. Ravon, Y. Schuurman, C. Mirodatos, D. Dubbeldam, H. Frost and R. Q. Snurr, Langmuir, 2009, 25, 7383.

70 I. Smallwood, Handbook of organic solvent properties, Butterworth-Heinemann, 2012.

71 F. Tian, X. Zhang and Y. Chen, RSC Adv., 2016, 6, 31214.

72 R. Numaguchi, H. Tanaka, S. Watanabe and M. T. Miyahara, J. Chem. Phys., 2013, 138, 054708.
73 G. A. Miller and R. B. Bernstein, J. Phys. Chem., 1959, 63, 710.

74 T. R. Welberry and T. Weber, Crystallogr. Rev., 2016, 22, 2.

75 P. S. Petkov, V. Bon, C. L. Hobday, A. B. Kuc, P. Melix, S. Kaskel, T. Düren and T. Heine, Phys. Chem. Chem. Phys., 2019, 21, 674.

76 N. Klein, C. Herzog, M. Sabo, I. Senkovska, J. Getzschmann, S. Paasch, M. R. Lohe, E. Brunner and S. Kaskel, Phys. Chem. Chem. Phys., 2010, 12, 11778.

77 S. M. Nathalie, B. Marie-Claire, L. Christophe, F. Isabelle, H. Pascal and V. Alain, Z. Anorg. Allg. Chem., 2016, 642, 709.

78 S. A. Sapchenko, D. G. Samsonenko, D. N. Dybtsev, M. S. Melgunov and V. P. Fedin, Dalton Trans., 2011, 40, 2196.

79 Z. Hu, S. Pramanik, K. Tan, C. Zheng, W. Liu, X. Zhang, Y. J. Chabal and J. Li, Cryst. Growth Des., 2013, 13, 4204.

80 R. N. Devi, M. Edgar, J. Gonzalez, A. M. Z. Slawin, D. P. Tunstall, P. Grewal, P. A. Cox and P. A. Wright, J. Phys. Chem. B, 2004, 108, 535.

81 C. Hyungphil, D. N. Dybtsev, K. Hyunuk and K. Kimoon, Chem.-Eur. J., 2005, 11, 3521.

82 J.-L. Dong, D.-Z. Wang, Y.-Y. Jia and D.-H. Wang, J. Mol. Struct., 2017, 1142, 304.

83 S. A. Sapchenko, D. G. Samsonenko, D. N. Dybtsev and V. P. Fedin, Inorg. Chem., 2013, 52, 9702.

84 A. P. Nelson, D. A. Parrish, L. R. Cambrea, L. C. Baldwin, N. J. Trivedi, K. L. Mulfort, O. K. Farha and J. T. Hupp, Cryst. Growth Des., 2009, 9, 4588.

85 O. Alduhaish, H. Wang, B. Li, H. D. Arman, V. Nesterov, K. Alfooty and B. Chen, ChemPlusChem, 2016, 81, 764.

86 S. W. Lee, H. J. Kim, Y. K. Lee, K. Park, J.-H. Son and Y.-U. Kwon, Inorg. Chim. Acta, 2003, 353, 151.

87 L.-P. Hsu, J.-Y. Wu and K.-L. Lu, J. Inorg. Organomet. Polym. Mater., 2007, 17, 259.

88 H. Aggarwal, R. K. Das, P. M. Bhatt and L. J. Barbour, Chem. Sci., 2015, 6, 4986.

89 P. Kanoo, R. Matsuda, M. Higuchi, S. Kitagawa and T. K. Maji, Chem. Mater., 2009, 21, 5860.

90 H. Wang, J. Getzschmann, I. Senkovska and S. Kaskel, Microporous Mesoporous Mater., 2008, 116, 653.

91 T. F. Willems, C. H. Rycroft, M. Kazi, J. C. Meza and M. Haranczyk, Microporous Mesoporous Mater., 2012, 149, 134.

92 L. Sacconi and I. Bertini, J. Am. Chem. Soc., 1968, 90, 5443. 93 J. Ghannam, T. Al Assil, T. C. Pankratz, R. L. Lord, M. Zeller and W.-T. Lee, Inorg. Chem., 2018, 57, 8307.

94 K. Tan, N. Nijem, P. Canepa, Q. Gong, J. Li, T. Thonhauser and Y. J. Chabal, Chem. Mater., 2012, 24, 3153.

95 D. A. Guzonas and D. E. Irish, Can. J. Chem., 1988, 66, 1249. 96 A. Krylov, A. Vtyurin, P. Petkov, I. Senkovska, M. Maliuta, V. Bon, T. Heine, S. Kaskel and E. Slyusareva, Phys. Chem. Chem. Phys., 2017, 19, 32099.

97 L. Brillouin, Ann. Phys., 1922, 9, 88.

98 L.-M. Yang, P. Ravindran, P. Vajeeston and M. Tilset, RSC Adv., 2012, 2, 1618.

99 L. Zhu, X. Chen, Q. Zhao, Z. Li, X. Zhang and B. Sun, Z. Anorg. Allg. Chem., 2010, 636, 1441. 
100 Y. Pang, D. Tian, X.-F. Zhu, Y.-H. Luo, X. Zheng and H. Zhang, CrystEngComm, 2011, 13, 5142.

101 J. Zhao, Y. Wang, W. Dong, Y. Wu, D. Li, B. Liu and Q. Zhang, Chem. Commun., 2015, 51, 9479.

102 J. Vallejo, F. R. Fortea-Pérez, E. Pardo, S. Benmansour, I. Castro, J. Krzystek, D. Armentano and J. Cano, Chem. Sci., 2016, 7, 2286.
103 M. Kurmoo, Chem. Soc. Rev., 2009, 38, 1353.

104 L. Sacconi, Coord. Chem. Rev., 1972, 8, 351.

105 C. H. Hendon and A. Walsh, Chem. Sci., 2015, 6, 3674.

106 J. N. Harvey, Principles and applications of density functional theory in inorganic chemistry I, Springer, 2004, p. 151. 